\title{
Existence and decay rates for a semilinear dissipative fractional second order evolution equation
}

\author{
Ruy Coimbra Charão I; Jaqueline Luiza Horbach II
}

\begin{abstract}
In this work we study the existence and uniqueness of solutions and decay rates to the total energy and the $L^{2}$-norm of solution for a semilinear second order evolution equation with fractional damping term and under effects of a generalized rotational inertia term in the case of plate equation. This system also includes equations of Boussinesq type that model hydrodynamic problems. We show decay rates depend- ing on the fractional powers of the operators and using an asymptotic expansion of the solution to the linear problem, we prove for some cases depending on the exponents of the operators, the optimality of the decay rates.
\end{abstract}

Keywords: Plate/Boussinesq type equation; Fractional Laplacians; Generalized rotational inertia; Fractional dissipation; Existence and uniqueness; Decay rates. 


\section{INTRODUCTION}

We consider in this work the following Cauchy problem associated to plate/Boussinesq type equations with a fractional damping and a generalized fractional rotational inertia term in $\mathrm{R}^{n}$

$$
\left\{\begin{array}{l}
u_{t t}+(-\Delta)^{\delta} u_{t t}+\alpha \Delta^{2} u-\Delta u+(-\Delta)^{\theta} u_{t}=\beta(-\Delta)^{\gamma}\left(u^{p}\right) \\
u(0, x)=u_{0}(x) \\
u_{t}(0, x)=u_{1}(x)
\end{array}\right.
$$

with $u=u(t, x),(t, x) \in(0, \infty) \times R n, a>0, \beta \in R, p>1$ integers and $\mathrm{u} 0, \mathrm{u} 1$ are the initial date. The Laplacian power $\delta, \theta$ and $\mathrm{y}$ are such that $0 \leq \delta$ $\leq 2,0 \leq \theta \leq 2$ and $0 \leq y \leq 1$.

The function $u=u(x, t)$, for example $e$, in the case $\delta=1$ and $\beta=0$, describes the transverse displacement of a plate without non-linear effects, but subject to effects of rotational inertia and a fractional dissipation represented by the term $(-\Delta) \theta u t$. In the case $\delta=0$ and $\beta=0$ the linear equation in (1) models the plate displacement without rotational inertial effects.

In the case $\delta=2, \beta 6=0$ and $Y=1$ the equation in (1) is a Boussinesq equation of sixth order under dissipative effects to model hydrodynamic problems (see [16], [6]). If $\delta=a=0, Y=1, \beta 6=0$ and without the dissipative term the equation in (1) is a generalized Boussinesq equation. If the nonlinearity has the form $\Delta\left(\begin{array}{ll}u & 2\end{array}\right)$ the equation is called the Boussinesq equation $(\mathrm{Bq})$. With this type of nonlinearity and $\delta=1, \mathrm{a}=0$ and without the dissipative term, the equation in (1) is called the improved Boussinesq equation (IBq). This same equation with more general linearity as it appears above in (1) is called the IMBq equation (Modified IBq) (see [15]). All these variants of Boussinesq have many physical applications, such as the propagation of longitudinal waves of deformation in an elastic rod in the case of the dimension $\mathrm{n}=1$, propagation of shallow-water waves. Six-order Boussinesq equation was derived in the study of surface layers of nonlinear plasmas and non-linear chains (see [1], [5]). In Maugin [12], Maugin proposed such a Boussinesq model to model the dynamics of nonlinear networks in elastic crystals. 
In the article by Charão-Horbach-Ikehata [7] the authors studied the equation in (1) for the linear case $\beta=0$. In that paper they studied decay rates for the linear problem and showed that the rates are optimal under the conditions $12<\theta<\min \{32, \delta+12\}$ and $0<\delta<\theta$.

In this paper our aim is to show the existence and uniqueness of solution for both the linear problem and the semilinear problem and to get decay rates for the semilinear problem under suitable conditions on the initial data and the fractional exponents of the Laplacian operator. Our results improve several previous works (see [2], [3], [8], [9] [11], [13], [14], [15], [16]).

\subsection{Basic Results}

In this section we introduce some results and technical lemmas that will be used in this paper. Part of these results are known and standard and the proof is not necessary.

The method to prove our results such as properties of the Cauchy Problem (1) includes to apply the Fourier transform to get an equivalent Cauchy problem in Fourier space associated with the problem (1). Thus, we need to define the Fourier Transform of a function as usual.

Definition 1.1: If $u \in L^{2}\left(R^{n}\right)$ then we denote for Fu the Fourier Transform of u given by

$$
\hat{u}(\xi)=\mathcal{F} u(\xi)=\left(\frac{1}{2 \pi}\right)^{\frac{\dot{n}}{2}} \int_{\mathbb{R}^{n}} e^{-i x \cdot \xi} u(x) d x .
$$

In addition, we denote by $F^{-1} \hat{u}$ the inverse Fourier Transform of $\hat{u}$ given by

$$
\mathcal{F}^{-1} \hat{u}(\xi)=\left(\frac{1}{2 \pi}\right)^{\frac{n}{2}} \int_{\mathbb{R}^{n}} e^{i x \cdot \xi} \hat{u}(\xi) d \xi
$$

For $u \in H^{a}\left(R^{n}\right)$ the operator $(-\Delta)^{a}$ is defined via Fourier transform by

$$
(-\Delta)^{\alpha} u(x)=\mathcal{F}^{-1}\left[|\cdot|^{2 \alpha} \hat{u}(\cdot)\right](x), \quad x \in \mathbb{R}^{n}
$$


Theorem 1.1 (Plancherel Identity) For all function $u \in L^{2}\left(R^{n}\right)$ it holds that $\|u\|=\|F u\|$.

In this work we use the space $H^{s}\left(R^{n}\right)$ for $s \in R$. The following definition is the equivalent to the usual definition of $H^{s}\left(R^{n}\right)$.

Definition 1.2: For $s \in \mathrm{R}$ we define the space

$$
H^{s}\left(R^{n}\right)=\left\{u \in S^{\prime}\left(R^{n}\right) /\left(1+|\xi|^{2}\right)^{s / 2} \hat{u} \in L^{2}\left(R^{n}\right)\right\}
$$

In the case $H^{s}\left(\mathrm{R}^{n}\right)$ with $s>0$ we use the following equivalent norm and inner product

$$
\|u\|_{H^{s}}^{2}=\int_{\mathbb{R}^{n}}\left(1+|\xi|^{2 s}\right)|\hat{u}|^{2} d \xi \quad \text { and } \quad(u, v)_{H^{s}}=\int_{\mathbb{R}^{n}}\left(1+|\xi|^{2 s}\right) \hat{u} \overline{\hat{v}} d \xi .
$$

In the case $H^{-s}\left(\mathrm{R}^{n}\right)$ with $s>0$ we use the norm and inner product given by

$$
\|u\|_{H^{-s}}^{2}=\int_{\mathbb{R}^{n}}\left(1+|\xi|^{2 s}\right)^{-1}|\hat{u}|^{2} d \xi \quad \text { and } \quad(u, v)_{H^{-s}}=\int_{\mathbb{R}^{n}}\left(1+|\xi|^{2 s}\right)^{-1} \hat{u} \overline{\hat{v}} d \xi
$$

Remark 1.1: When $\mathrm{s}=2$ we consider the following norm and inner product equivalent to usual

$$
\|u\|_{H^{2}}^{2}=\int_{\mathbb{R}^{n}}\left(1+|\xi|^{2}+\alpha|\xi|^{4}\right)|\hat{u}|^{2} d \xi \quad \text { and } \quad(u, v)_{H^{2}}=\int_{\mathbb{R}^{n}}\left(1+|\xi|^{2}+\alpha|\xi|^{4}\right) \hat{u} \overline{\hat{v}} d \xi .
$$

Let consider the space of functions where we only take in account the derivative of greater order, that is, the space $\dot{W}^{m, p}\left(\mathrm{R}^{n}\right)$, is defined for $m, p$ $\in Z, p \geq 1$ by

$$
\dot{W}^{m, p}\left(\mathbb{R}^{n}\right)=\left\{u \in \mathcal{S}^{\prime}\left(\mathbb{R}^{n}\right) / \exists f \in L^{p}\left(\mathbb{R}^{n}\right) \text { with } u=(-\Delta)^{-m / 2} f\right\}, \quad \text { for all } \quad m \in \mathbb{Z}, p \in \mathbb{Z}, p \geq 1 .
$$

We may represent this space by

$$
\dot{W}^{m, p}\left(\mathbb{R}^{n}\right)=(-\Delta)^{-m / 2} L^{p}\left(\mathbb{R}^{n}\right)
$$


The norm in this space is defined by

$$
\|u\|_{\dot{W}^{m, p}}=\int_{\mathbb{R}^{n}}\left|(-\Delta)^{m / 2} u(x)\right|^{p} d x
$$

Using the inner product and norm defined above, we can show some properties involving the spaces $H^{s}\left(R^{n}\right)$. These properties have fundamental importance to show existence and uniqueness of solution for both linear and semilinear case.

Lemma 1.1: Let $u \in H^{s}\left(R^{n}\right)$. If $s>\frac{n}{2}$ then exist a constant $C>0$ such that

$$
|u(x)| \leq C\|u\|_{H^{s}}, \quad \forall x \in \mathbb{R}^{n}
$$

That is, when $s>\frac{n}{2}$ we have $H^{s}\left(\mathrm{R}^{n}\right)$ continuously imbedding in $L^{\infty}\left(\mathrm{R}^{n}\right)$.

Lemma 1.2: $\left(H^{s}\left(\mathrm{R}^{n}\right)\right.$ is an algebra, $\left.s>n / 2\right)$ Let $u, w \in H^{s}\left(\mathrm{R}^{n}\right)$. If $s>\frac{n}{2}$ then exist a constant $\mathrm{C}>0$ such that

$$
\|u w\|_{H^{s}} \leq C\|u\|_{H^{s}}\|w\|_{H^{s}}
$$

This Lemma is proved in the article of Kato-Ponce and Wang-Chen.

Lemma 1.3: Let $u \in \mathrm{H}^{\mathrm{s}}\left(\mathrm{R}^{\mathrm{n}}\right)$. If and $p \geq 1$ integer then there exist $a$ constant $\mathrm{C}>0$ such that

$$
\left\|u^{p}\right\|_{H^{s}} \leq C\|u\|_{H^{s}}^{p} .
$$

Proof: For $p=1$ the lemma is trivial. For $p>1$ integer applying Lemma $1.2 p-1$ times we get the result.

Lemma 1.4: Let $u \in H^{s}\left(\mathrm{R}^{n}\right)$. If $s>\frac{n}{2}$ and $p>1$ integer then exist a constant $\mathrm{C}>0$ such that 
$\left\|u^{p}\right\|_{L^{1}} \leq C\|u\|_{H^{s}}^{p}$

Proof: definition of norm $L^{1}\left(R^{n}\right)$ we have $\left\|u^{p}\right\|_{L^{1}}=\int_{\mathbb{R}^{n}}\left|u^{p}\right| d x \leq \int_{\mathbb{R}^{n}}\left|u^{p-1}\right||u| d x$.

Using Hölder's inequality with ${ }^{\frac{1}{2}+\frac{1}{2}}=1$ we have

$$
\left\|u^{p}\right\|_{L^{1}} \leq\left(\int_{\mathbb{R}^{n}}\left|u^{p-1}\right|^{2} d x\right)^{1 / 2}\left(\int_{\mathbb{R}^{n}}|u|^{2} d x\right)^{1 / 2} \leq\left\|u^{p-1}\right\|_{L^{2}}\|u\|_{L^{2}} \leq\left\|u^{p-1}\right\|_{H^{s}}\|u\|_{H^{s}} .
$$

The proof follows from the fact that $p>1$ is integer and $H^{s}\left(\mathrm{R}^{n}\right)$ is an algebra for $s>\frac{n}{2}$

Lemma 1.5: Let $u, \mathrm{w} \in \mathrm{H}^{\mathrm{s}}\left(\mathrm{R}^{\mathrm{n}}\right), s>\frac{n}{2}$ and $p>1$ integer. Then there exist a constant $\mathrm{C}>0$ such that

$$
\left\|u^{p}-w^{p}\right\|_{H^{s}} \leq C\left(\|u\|_{H^{s}}^{p-1}+\|w\|_{H^{s}}^{p-1}\right)\|u-w\|_{H^{s}} .
$$

Proof: Define $h(\lambda)=\lambda^{p}$ then $h^{1}(\lambda)=p \lambda^{p-1}$. By the Mean Value Theorem we have

$$
u^{p}-w^{p}=p \lambda^{p-1}(u-w)
$$

where

$$
\lambda=(1-\epsilon) u+\epsilon w,
$$

for some $0<\in<1$.

Therefore, using Lemma 1.1 and the fact that $p$ is integer, we have $\left\|u^{p}-w^{p}\right\|_{H^{s}}=p\left\|\lambda^{p-1}(u-w)\right\|_{H^{s}} \leq C\|\lambda\|_{H^{s}}^{p-1}\|u-w\|_{H^{s}} \leq C\left(\|u\|_{H^{s}}^{p-1}+\|w\|_{H^{s}}^{p-1}\right)\|u-w\|_{H^{s}}$. 
1.1.1 Abstract Linear Problem: Existence of Solution

Let $X$ be a Banach space and $B$ a linear operator on $X$. Considering the abstract Cauchy problem

$$
\left\{\begin{array}{l}
\frac{d U}{d t}=B U(t) \quad t>0 \\
U(0)=U_{0}
\end{array}\right.
$$

the following result holds.

Theorem 1.2: If $B$ is the infinitesimal generator of a Co-semigroup on $X$ then, for each $U_{0} \in D(B)$ the problem (2) admits a unique strong solution

$$
U(t)=S(t) U_{0} \in C\left(\mathbb{R}^{+}, D(B)\right) \cap C^{1}\left(\mathbb{R}^{+}, X\right),
$$

where $S(t)$ is the semigroup generated by the operator $B$.

If $U_{0} \in X$ then we say that $U(t)=S(t) U_{0} \in C\left(R^{+}, X\right)$ is a weak solution for (2).

Theorem 1.3: If $B$ is the infinitesimal generator of a Co-semigroup contractions on a Banach space $X$ and $J$ is a linear and bounded operator on $X$, then $B+J$ is a infinitesimal generator of $C_{0}$-semigroup on $X$.

\subsubsection{Abstract Semilinear Problem: Existence of Solution}

Let $X$ be a Banach space and $B$ a linear operator on $X$. Consider the abstract Cauchy problem

$$
\left\{\begin{array}{l}
\frac{d U}{d t}=B U(t)+F U(t) \\
U(0)=U_{0}
\end{array}\right.
$$

where $U_{0} \in X, t>0$ and $F$ is a nonlinear operator.

Definition 1.3: An operator $F: D(B) \rightarrow D(B)$ is continuous Lipschitz on bounded sets of $D(B) \subset X$ if given a constant $M>0$ there exist a constant $L_{M}>$ 0 such that 


$$
\|F(U)-F(W)\|_{X}+\|B(F(U)-F(W))\|_{X} \leq C L_{M}\left(\|U-W\|_{X}+\|B(U-W)\|_{X}\right)
$$

for all $U$ and $W$ in $D(B)$ such that

$$
\|U\|_{X}+\|B U\|_{X} \leq M \text { and }\|W\|_{X}+\|B W\|_{X} \leq M
$$

The next result is well known.

Theorem 1.4: Let $F: D(B) \rightarrow D(B)$ a continuous Lipschitz operator on bounded sets of $D(B) \subset X$. Then, for each $U_{0} \in D(B)$, there exist a unique strong solution $U=U(t)$ of the Cauchy Problem (3) defined in a maximal interval $\left[0, T_{m}\right)$ such that only one of the following conditions is true
(i) $T_{m}=\infty$
(ii) $T_{m}<\infty$ and $\lim _{t \rightarrow T_{m}}\|U\|_{X}+\|B U\|_{X}=+\infty$.

The solution $U=U(t)$ of Cauchy Problem (3) belongs to the following class

$$
U \in C^{1}\left(\left[0, T_{m}\right), X\right) \cap C\left(\left[0, T_{m}\right), D(B)\right) .
$$

\subsubsection{Tecnical Lemmas}

In this section we present some lemmas that we use to prove the existence and uniqueness of solution as well as some lemmas used toget decay rates in time of the solution. Some of those lemmas have already been proved in HORBACH, J. L., IKEHATA, R. e CHARÃO, R. C.

Lemma 1.6: Let $c$ and $r$ be positive numbers and $a \in R$. Then, there exists a constant $C>0$ such that

$$
t^{r} e^{-c|\xi|^{a} t} \leq C|\xi|^{-a r} \quad \forall t>0, \xi \in \mathbb{R}^{n}, \xi \neq 0
$$

Lemma 1.7: Let $k>-n, \theta>0$ and $C>0$. Then there existe $a$ constant $K>O$ depending on $n$ such that 


$$
\int_{\mathbb{R}^{n}} e^{-C|\xi|^{\vartheta} t}|\xi|^{k} d \xi \leq K t^{-\frac{n+k}{\vartheta}}, \quad \forall t>0
$$

Lemma 1.8: Let $k>-n, \theta>0$ and $C>0$. Then there exist $a$ constant $K>0$ depending on $n$ such that

$$
\int_{\mathbb{R}^{n}} e^{-C|\xi|^{\vartheta} t}|\xi|^{k} d \xi \leq K(1+t)^{-\frac{n+k}{\vartheta}}, \quad \forall t>0 .
$$

Lemma 1.9: Let $n \geq 1, a>1$ and $p>1$ integer. Then, there exist a constant $C=C(a, p)>0$ such that

$$
(1+t)^{a} \int_{0}^{t}(1+\tau)^{-p a}(1+t-\tau)^{-a} d \tau \leq C, \quad \forall t>0
$$

Proof: To estimate the above integral, we separate it into two integrals, that is, an integral over the interval $\left[0, \frac{t}{2}\right]$ and the other over $\left[\frac{t}{2}, t\right]$.

First, we observe that, if $0 \leq T \leq \frac{t}{2}$ we have $1+t \leq 1+2 t-t \leq$ $2+2 t-2 T \leq 2(1+t-T)$ and this implies $(1+t-T)^{-a} \leq 2 a(1+t)^{-a}$, for $a>$ 1. Then, for $a p>1$, we get

$$
\begin{aligned}
& (1+t)^{a} \int_{\frac{t}{2}}^{t}(1+\tau)^{-p a}(1+t-\tau)^{-a} d \tau \leq 2^{a p}(1+t)^{a-a p} \int_{\frac{t}{2}}^{t}(1+t-\tau)^{-a} d \tau \\
& \leq-\left.2^{a p}(1+t)^{a-a p} \frac{(1+t-\tau)^{1-a}}{1-a}\right|_{\frac{t}{2}} ^{t} \leq 2^{a p}(1+t)^{a-a p} \frac{1}{a-1} \leq \frac{2^{a p}}{a-1} .
\end{aligned}
$$

Finally, we define

$$
C(a, p)=\max \left\{\frac{2^{a p}}{a-1}, \frac{2^{a}}{a p-1}\right\}
$$

to get the proof of lemma for all $t>0$. 


\section{EXISTENCE AND UNIQUENESS: LINEAR PROBLEM}

In this section using the the semigroup theory we show the existence and uniqueness of solution to the following Cauchy problem associated with an equation of Boussines/plate type with a structural rotational inertia (to the case of plates) and a fractional dissipation in $\mathrm{R}^{n}$ with $n \geq 1$.

$$
\left\{\begin{array}{l}
u_{t t}+(-\Delta)^{\delta} u_{t t}+\alpha \Delta^{2} u-\Delta u+(-\Delta)^{\theta} u_{t}=0 \\
u(0, x)=u_{0}(x) \\
u_{t}(0, x)=u_{1}(x)
\end{array}\right.
$$

where $u=u(t, x),(t, x) \in(0,) \mathrm{R}^{n}, a>0$ is a constant. The exponents of the Laplacian operators $\delta$ and $\theta$ are such that $0 \leq \delta \leq 2$ and $0 \leq \theta \leq$ $\frac{2+\delta}{2}$.

Formally, the inner product in $L^{2}\left(R^{n}\right)$ between the differential equation in (4) with $u_{t}$ is given by

$$
\frac{1}{2} \frac{d}{d t} E(t)+\left\|(-\Delta)^{\theta / 2} u_{t}\right\|^{2}=0, \quad \forall t>0
$$

where the total energy $E(t)$ ) of system (4) is given by

$$
E(t)=\frac{1}{2}\left(\left\|u_{t}\right\|^{2}+\left\|(-\Delta)^{\delta / 2} u_{t}\right\|^{2}+\alpha\|\Delta u\|^{2}+\|\nabla u\|^{2}\right) .
$$

Then, isnatural to define the energy space as

$$
X=H^{2}\left(\mathbb{R}^{n}\right) \times H^{\delta}\left(\mathbb{R}^{n}\right) .
$$

Note that in case $\delta>2$ we have $H^{2}\left(\mathrm{R}^{n}\right) \subset H^{\delta}\left(\mathrm{R}^{n}\right)$ and this is unnatural because in this case $u_{t}$ would be more regular than $u$. Another relationship we need to take care is the relationship between $\delta$ and $\theta$ because in the energy identity appears $(-\Delta)^{\theta / 2} u_{t}$ and in the case $\delta \leq \theta$ we have $H^{\theta}\left(R^{n}\right) \subset$ $H^{\delta}\left(\mathrm{R}^{n}\right)$. Also it is necessary to consider the relationship that comes from 
Luz-Ikehata-Charão (see [4]) where the condition of $\theta \leq \frac{2+\delta}{2}$ appears. To show the existence and uniqueness of solution we need consider two case between $\delta$ and $\theta$ and we rewrite the Problem (4) in a problem of first order on $X$ as follows

$$
\left\{\begin{array}{l}
\frac{d U}{d t}=B U+J(U) \\
U(0)=U_{0}
\end{array}\right.
$$

where $U=\left(u, u_{t}\right), U(0)=\left(u_{0}, u_{1}\right)$ and the operators $B$ and $J$ depends on the cases $\theta<\delta$ and $\theta \geq \delta$.

Before we show the existence and uniqueness we need the definition of two important operators, the operators $A_{2}$ and $A_{\theta}$. These operators are essential for the definition of the operator $B$. For the case $0 \leq \theta<\delta$ we use the operator $A_{2}$ to define $B$, while in the case $0 \leq \delta \leq \theta$ we use the two operators, $A_{2}$ and $A_{\theta}$ to define the operator $B$.

\subsection{The operator $A_{\mathrm{j}}$}

To define the operator $A_{j}$ we need to consider $j \geq \delta$. We define the domain of $A_{j}$ as the subspace of $H\left(\mathrm{R}^{n}\right)$ given by 2

$$
\begin{aligned}
& D\left(A_{j}\right)=\left\{v \in H^{j}\left(\mathbb{R}^{n}\right) / \exists z=z_{v} \in H^{\delta}\left(\mathbb{R}^{n}\right) \quad\right. \text { such that } \\
& \left.\left((-\Delta)^{j / 2} v,(-\Delta)^{j / 2} \psi\right)+(v, \psi)=(z, \psi)+\left((-\Delta)^{\delta / 2} z,(-\Delta)^{\delta / 2} \psi\right), \forall \psi \in H^{j}\left(\mathbb{R}^{n}\right)\right\} .
\end{aligned}
$$

Following the definition of $D\left(A_{j}\right)$ the operator $A_{j}$, it shall be defined as

$$
\begin{gathered}
A_{j}: D\left(A_{j}\right) \longrightarrow H^{\delta}\left(\mathbb{R}^{n}\right) \\
A_{j} v=z_{v}, \quad v \in D\left(A_{j}\right) .
\end{gathered}
$$

Formally we have that the operator $A_{j}$ is given by

$$
A_{j}=\left(I+(-\Delta)^{\delta}\right)^{-1}\left(I+(-\Delta)^{j}\right) \text {. }
$$


Lemma 2.1: For all $v \in \mathrm{Hi}^{\mathrm{j}}\left(\mathrm{R}^{\mathrm{n}}\right)$ exist at most one $\mathrm{y}=\mathrm{y}_{\mathrm{v}} \in \mathrm{H}^{\delta}\left(\mathrm{R}^{\mathrm{n}}\right)$ such that

$$
\left((-\Delta)^{j / 2} v,(-\Delta)^{j / 2} \psi\right)+(v, \psi)=(z, \psi)+\left((-\Delta)^{\delta / 2} z,(-\Delta)^{\delta / 2} \psi\right), \quad \forall \psi \in H^{j}\left(\mathbb{R}^{n}\right)
$$

Proof: If $y_{1}, y_{2} \in H^{\delta}\left(R^{n}\right)$ satisfy the relation (9) and because $C_{0}^{\infty}\left(R^{n}\right)$ is dense in $H\left(\mathrm{R}^{n}\right)$ we have

$$
\left(y_{1}-y_{2}, \psi\right)+\left((-\Delta)^{\delta / 2}\left(y_{1}-y_{2}\right),(-\Delta)^{\delta / 2} \psi\right)=0, \quad \forall \psi \in C_{0}^{\infty}\left(\mathbb{R}^{n}\right)
$$

Consider $\quad y:=y_{1}-y_{2}$, by the density of $C_{0}^{\infty}\left(R^{n}\right)$ in $H^{\delta}\left(R^{n}\right)$, there exist $\left\{\psi_{v}\right\}_{v \in \mathrm{N}} \in C_{0}^{\infty}\left(\mathrm{R}^{n}\right)$ such that

$\lim _{\nu \longrightarrow \infty} \psi_{\nu}=y$ in $H^{\delta}\left(\mathbb{R}^{n}\right)$

therefore,

$\left\|\psi_{\nu}-y\right\|_{H^{\delta}} \longrightarrow 0$, if $\nu \longrightarrow \infty$

or yet

$\left\|\psi_{\nu}-y\right\|_{H^{\delta}}^{2}=\|y\|_{H^{\delta}}^{2}-2\left(y, \psi_{\nu}\right)_{H^{\delta}}+\left\|\psi_{\nu}\right\|_{H^{\delta}}^{2} \longrightarrow 0, \quad$ when $\quad \nu \longrightarrow \infty$.

Due to

$\left|\left\|\psi_{\nu}\right\|-\|y\|\right| \leq\left\|\psi_{\nu}-y\right\|$

we conclude that

$\left\|\psi_{\nu}\right\|_{H^{\delta}} \longrightarrow\|y\|_{H^{\delta}} \quad$ in case $\quad \nu \longrightarrow \infty$.

Using (11) and (12) we conclude that

$$
\lim _{\nu \longrightarrow \infty}\left(y, \psi_{\nu}\right)_{H^{\delta}}=\|y\|_{H^{\delta}}^{2}
$$

From (10) and the definition of inner product in $H^{\delta}\left(\mathrm{R}^{n}\right)$ we have 
$0=\left(y, \psi_{\nu}\right)+\left((-\Delta)^{\delta / 2} y,(-\Delta)^{\delta / 2} \psi_{\nu}\right)=\left(y, \psi_{\nu}\right)_{H^{\delta}}$.

Thus, from (11) and (12) we have

$$
0=\lim _{\nu \rightarrow \infty}\left(y, \psi_{\nu}\right)_{H^{\delta}}=\|y\|_{H^{\delta}}^{2}
$$

Therefore, we conclude thar $y_{1}=y_{2}$.

Remark 2.1: Due to $v \equiv 0 \in D\left(A_{j}\right)$ and Lemma 2.1 it follows that $A_{j}$ is well defined.

Lemma 2.2: For $j \geq \delta \geq 0$ it holds that $D\left(A_{j}\right) H^{2 j-\delta}\left(R^{n}\right)$ and there exist a constant $C>0$ such that

$$
\|v\|_{H^{2 j-\delta}} \leq C\left\|A_{j} v\right\|_{H^{\delta}}, \quad \forall v \in D\left(A_{j}\right) .
$$

Proof: Let $v \in D\left(A_{j}\right)$ for definition of $D\left(A_{j}\right)$, there exist $y=y_{v} \in H^{\delta}\left(\mathrm{R}^{n}\right)$ such that

$$
\left((-\Delta)^{j / 2} v,(-\Delta)^{j / 2} \psi\right)+(v, \psi)=(z, \psi)+\left((-\Delta)^{\delta / 2} z,(-\Delta)^{\delta / 2} \psi\right), \quad \forall \psi \in H^{j}\left(\mathbb{R}^{n}\right) .
$$

We now define the functional $F_{1}: H^{\delta}\left(\mathrm{R}^{n}\right) \rightarrow \mathrm{R}$ by

$$
\left\langle F_{1}, \psi\right\rangle=(y, \psi)+\left((-\Delta)^{\delta / 2} y,(-\Delta)^{\delta / 2} \psi\right), \quad \forall \psi \in H^{\delta}\left(\mathbb{R}^{n}\right) .
$$

It is easy to see that $F_{1}$ is well define and linear. Moreover, using Plancherel theorem 1.1 and the norm define in $H^{\delta}\left(R^{n}\right)$ we proof that $F_{1}$ is a bounded operator. In fact

$$
\begin{aligned}
\left|\left\langle F_{1}, \psi\right\rangle\right| & \leq|(y, \psi)|+\left|\left((-\Delta)^{\delta / 2} y,(-\Delta)^{\delta / 2} \psi\right)\right| \leq\|y\|\|\psi\|+\left\|(-\Delta)^{\delta / 2} y\right\|\left\|(-\Delta)^{\delta / 2} \psi\right\| \\
& \leq\|\hat{y}\|\|\hat{\psi}\|+\left\||\xi|^{\delta} \hat{y}\right\|\left\||\xi|^{\delta} \hat{\psi}\right\| \leq 2\|y\|_{H^{\delta}}\|\psi\|_{H^{\delta}}, \quad \forall \psi \in H^{\delta}\left(\mathbb{R}^{n}\right) .
\end{aligned}
$$

Using the density of $\mathrm{S}\left(\mathrm{R}^{n}\right)$ in $H\left(\mathrm{R}^{n}\right)$, the varational problem (13) takes the following form

$$
\left((-\Delta)^{j / 2} v,(-\Delta)^{j / 2} \psi\right)+(v, \psi)=\left\langle F_{1}, \psi\right\rangle, \quad \forall \psi \in \mathcal{S}\left(\mathbb{R}^{n}\right) .
$$


Thus we conclude that $(-\Delta)^{j} v+v=F_{1}$ in $S^{\prime}\left(R^{n}\right)$. Applying the Fourier Transform, where $z=A_{j} v$, we conclude

$$
\hat{z}=\widehat{A_{j} v}=\frac{1+|\xi|^{2 j}}{1+|\xi|^{2 \delta}} \hat{v}
$$

Calculating the $L^{2}\left(R^{n}\right)$ norm for each term in the identity (14) we obtain

$$
\int_{\mathbb{R}^{n}}\left(1+|\xi|^{2 \delta}\right)^{-1}\left(1+|\xi|^{2 j}\right)^{2}|\hat{v}|^{2} d \xi=\int_{\mathbb{R}^{n}}\left(1+|\xi|^{2 \delta}\right)|\hat{z}|^{2} d \xi
$$

From the fact that $\left(1+|\xi|^{2 \delta}\right)^{-1}\left(1+|\xi|^{2 j}\right)^{2}$ is equivalent to $1+$ $\mid \xi^{2(2 j-\delta)}$, we conclude that

$$
\int_{\mathbb{R}^{n}}\left(1+|\xi|^{2(2 j-\delta)}\right)|\hat{v}|^{2} d \xi \leq C \int_{\mathbb{R}^{n}}\left(1+|\xi|^{2 \delta}\right)|\hat{z}|^{2} d \xi
$$

Following (15) we have that

$$
\|v\|_{H^{2 j-\delta}} \leq C\|z\|_{H^{\delta}}=C\left\|A_{j} v\right\|_{H^{\delta}}
$$

for all $v \in D\left(A_{j}\right)$.

Note that the condition of $\delta \leq j$ is required, since $H^{2 j-\delta}\left(\mathrm{R}^{n}\right)$ must be contained in $H\left(\mathrm{R}^{n}\right)$.

Lemma 2.3: Let $0 \leq \delta \leq j$ then $\mathrm{H}^{2 j-\delta}\left(\mathrm{R}^{\mathrm{n}}\right) \subseteq \mathrm{D}\left(\mathrm{A}_{\mathrm{j}}\right)$, that is, Let $v \in$ $\mathrm{H}^{2 \mathrm{j}-\delta}\left(\mathrm{R}^{\mathrm{n}}\right)$ then there exist $y \in \mathrm{H}^{\delta}\left(\mathrm{R}^{\mathrm{n}}\right)$ such that

$$
\left((-\Delta)^{j / 2} v,(-\Delta)^{j / 2} \psi\right)+(v, \psi)=(z, \psi)+\left((-\Delta)^{\delta / 2} z,(-\Delta)^{\delta / 2} \psi\right), \quad \forall \psi \in H^{j}\left(\mathbb{R}^{n}\right) .
$$

Proof: Let $v \in H^{2 j-\delta}\left(\mathrm{R}^{n}\right)$ and $G_{1}: H^{\delta}\left(\mathrm{R}^{n}\right) \rightarrow \mathrm{R}$ given by

$$
\left\langle G_{1}, \psi\right\rangle=\left((-\Delta)^{j-\delta / 2} v,(-\Delta)^{\delta / 2} \psi\right)+(v, \psi), \quad \forall \psi \in H^{\delta}\left(\mathbb{R}^{n}\right) .
$$


Thus $G_{1}$ is well define and linear. Similarly to the proof that $F_{1}$ is continuous we may prove that $G_{1}$ is continuous $\left(\left|G_{1}\right| \leq 2 \backslash \nu \lambda_{H 2 j-\delta}\right)$.

Let $a_{1}: H^{\delta}\left(\mathrm{R}^{n}\right) \times H^{\delta}\left(\mathrm{R}^{n}\right) \rightarrow \mathrm{R}$, such that $a_{1}(\phi, \psi)=(\phi, \psi)+$ $\left((-\Delta)^{\delta / 2} \phi,(-\Delta)^{\delta / 2} \psi\right)$ for all $\psi, \phi \in H^{\delta}\left(\mathrm{R}^{n}\right)$.

We have that $a_{1}\left({ }^{\prime},{ }^{\prime}\right)$ is well defined and bilinear. Moreover $a_{1}\left({ }^{\prime},{ }^{\prime}\right)$ is continuous and coercive for all $\varphi, \psi \in H^{\delta}\left(\mathrm{R}^{n}\right)$, because

$$
\left|a_{1}(\varphi, \psi)\right| \leq 2\|\varphi\|_{H^{\delta}}\|\psi\|_{H^{\delta}}
$$

and

$$
a_{1}(\varphi, \varphi)=\|\varphi\|_{H^{\delta}}^{2}
$$

Therefore, the variational problem can be rewrite as

$$
a_{1}(y, \psi)=\left\langle G_{1}, \psi\right\rangle, \quad \forall \psi \in H^{\delta}\left(\mathbb{R}^{n}\right)
$$

From the Lax-Milgram Lemma the problem (17) admits unique solution $y=y_{v} \in H^{\delta}\left(R^{n}\right)$.

In particular (17) is valid for each $\psi \in\left(R^{n}\right)$, there exists only one $y$ $\in H^{\delta}\left(\mathrm{R}^{n}\right)$ such that

$$
(y, \psi)+\left((-\Delta)^{\delta / 2} y,(-\Delta)^{\delta / 2} \psi\right)=\left((-\Delta)^{j / 2} v,(-\Delta)^{j / 2} \psi\right)+(v, \psi), \quad \forall \psi \in \mathcal{D}\left(\mathbb{R}^{n}\right) .
$$

Using the density of $\mathrm{D}\left(\mathrm{R}^{n}\right)$ in $H\left(\mathrm{R}^{n}\right)$, by definition it follows that $v$ $\in D\left(A_{j}\right)$

Remark 2.2: The Lemmas 2.2 and 2.3 they says $D\left(A_{j}\right)=H^{2 j-\delta}$ $\left(\mathrm{R}^{\mathrm{n}}\right)$. When $0 \leq \delta \leq \theta$ we have $\delta \leq \theta \leq 2 \theta-\delta$ then $\mathrm{H}^{2 \theta-\delta}\left(\mathrm{R}^{\mathrm{n}}\right) \subset \mathrm{H}^{\theta}\left(\mathrm{R}^{\mathrm{n}}\right) \subset$ $\mathrm{H}^{\delta}\left(\mathrm{R}^{\mathrm{n}}\right)$. When $\mathrm{j}=2$ we consider $A_{2}$ given by

$$
\begin{aligned}
& A_{2}: D\left(A_{2}\right) \longrightarrow H^{\delta}\left(\mathbb{R}^{n}\right) \\
& A_{2}=\left(I+(-\Delta)^{\delta}\right)^{-1}\left(\alpha \Delta^{2}-\Delta+I\right) .
\end{aligned}
$$


The assumption $0 \leq \delta \leq 2$ implies that $\delta \leq 2 \leq 4-\delta$ then $\mathrm{H}^{4-\delta}\left(\mathrm{R}^{\mathrm{n}}\right) \subset$ $H^{2}\left(R^{n}\right) \subset H^{\delta}\left(R^{n}\right)$.

Then, similar to the case $j \geq \delta$, we can see that $D\left(A_{2}\right)=$ $H^{4-\delta}\left(R^{n}\right)$.

\subsection{Case $0 \leq \theta<\delta$ and $0 \leq \delta \leq 2$}

We rewrite the system (4) in matrix form, with $U=(u, v) \in X, U_{0}$ $=\left(u_{0}, u_{1}\right) \in X$,

$$
\left\{\begin{array}{l}
\frac{d U}{d t}=B_{1} U+J_{1}(U) \\
U(0)=U_{0}
\end{array}\right.
$$

where the operator $B_{1}: H^{4-\delta}\left(\mathrm{R}^{n}\right) \times H^{2}\left(\mathrm{R}^{n}\right) \rightarrow X$ and $J_{1}: X \rightarrow X$ are given by

$$
B_{1}=\left(\begin{array}{ll}
0 & I \\
-A_{2} & 0
\end{array}\right) \quad \text { and } \quad J_{1}(U)=\left(\begin{array}{l}
0 \\
\left(I+(-\Delta)^{\delta}\right)^{-1}\left(u-(-\Delta)^{\theta} v\right)
\end{array}\right) .
$$

Lemma 2.4: The operator $B_{1}$ is infinitesimal generator of contraction semigroup of class $C_{0}$ in $X$.

Proof: We proof that $B_{1}$ satisfies the hypotheses of Lumer-Phillips Theorem from semi-groups theory.

Let $(u, v) \in D\left(B_{1}\right)=H^{A-\delta}\left(\mathrm{R}^{n}\right) \times H^{2}\left(\mathrm{R}^{n}\right)$.

To proof that $B_{1}$ is dissipative we calculate the inner product

$$
\begin{aligned}
& \left(B_{1}(u, v),(u, v)\right)_{H^{2} \times H^{\delta}}=\operatorname{Re}\left(\left(v,-A_{2} u\right),(u, v)\right)_{H^{2} \times H^{\delta}}=(v, u)_{H^{2}}+\left(-A_{2} u, v\right)_{H^{\delta}} \\
& =\int_{\mathbb{R}^{n}}\left(1+|\xi|^{2}+\alpha|\xi|^{4}\right) \hat{v} \overline{\hat{u}} d \xi-\int_{\mathbb{R}^{n}}\left(1+|\xi|^{2 \delta}\right) \widehat{A_{2} u} \overline{\hat{v}} d \xi \\
& =\int_{\mathbb{R}^{n}}\left(1+|\xi|^{2}+\alpha|\xi|^{4}\right) \hat{v} \overline{\hat{u}} d \xi-\int_{\mathbb{R}^{n}}\left(1+|\xi|^{2 \delta}\right) \frac{1+|\xi|^{2}+\alpha|\xi|^{4}}{1+|\xi|^{2 \delta}} \hat{u} \hat{\hat{v}} d \xi \\
& =\int_{\mathbb{R}^{n}}\left(1+|\xi|^{2}+\alpha|\xi|^{4}\right)(\hat{v} \overline{\hat{u}}-\hat{u} \overline{\hat{v}}) d \xi=\int_{\mathbb{R}^{n}}\left(1+|\xi|^{2}+\alpha|\xi|^{4}\right) 2 i \operatorname{Img}(\hat{v} \overline{\hat{u}}) d \xi=0,
\end{aligned}
$$

because, 


$$
\widehat{A_{2} u}=\frac{1+|\xi|^{2}+\alpha|\xi|^{4}}{1+|\xi|^{2 \delta}} \hat{u}
$$

According to the definition $A_{2}$. Here $\operatorname{Img}(\hat{v}, \overline{\hat{u}})$ represents the imaginary part of $\hat{v} \bar{u}$ and $i=\sqrt{ }-1$. Taking the real part of $B_{1}(u, v),(u, v)$ $H^{2} \times H^{\delta}$ we get that $B_{1}$ is dissipative.

Now we show that $\operatorname{Im}\left(I-B_{1}\right)=H^{2}\left(\mathrm{R}^{n}\right) \times H^{\delta}\left(\mathrm{R}^{n}\right)$. It easy to prove that $\operatorname{Im}\left(I-B_{1}\right) \subset$ $H^{2}\left(\mathrm{R}^{n}\right) \times H^{\delta}\left(\mathrm{R}^{n}\right)$. We need to see that $H^{2}\left(\mathrm{R}^{n}\right) \times H^{\delta}\left(\mathrm{R}^{n}\right) \subset \operatorname{Im}\left(I-B_{1}\right)$. Let $(f, g) \in$ $H^{2}\left(\mathrm{R}^{n}\right) \times H^{\delta}\left(\mathrm{R}^{n}\right)$, then we prove that there exist $(u, v) \in D\left(B_{1}\right)$ such that $\left(I-B_{1}\right)(u, v)=$ $(f, g)$. Equivalently, by the definition of $B_{1}$, we need to prove that there exist $(u, v) \in D\left(B_{1}\right)$ such that $\left(u-v, v+A_{2} u\right)=(f, g)$

Thus, it is sufficient to show that there is $(u, v) \in \mathrm{D}\left(B_{1}\right)$ that satisfies

$$
\left\{\begin{array}{l}
u-v=f \\
v+A_{2} u=g
\end{array} .\right.
$$

Substituting the first equality $v=u-f$ in the second one, we have

$$
A_{2} u+u=g+f
$$

By using the Lax-Milgran lemma we can prove that there exist $u \in$ $H^{2}\left(R^{n}\right)$ satisfying the identity above. In particular we can obtain $A_{2} u+$ $u=g+f$ in $D^{\prime}\left(\mathrm{R}^{n}\right)$ where $u \in H^{2}\left(\mathrm{R}^{n}\right), g \in H^{\delta}\left(\mathrm{R}^{n}\right)$ and $f \in H^{2}\left(\mathrm{R}^{n}\right)$. Then applying the Fourier transform we can rewrite the identity above as follows

$$
\left(1+|\xi|^{2 \delta}\right)^{1 / 2} \widehat{A_{2} u}=\left(1+|\xi|^{2 \delta}\right)^{1 / 2}(\hat{f}+\hat{g}-\hat{u})
$$

Calculating the $L^{2}$-norm on each side of the above identity we have 


$$
\left\|A_{2} u\right\|_{H^{\delta}}^{2} \leq\|f\|_{H^{\delta}}^{2}+\|g\|_{H^{\delta}}^{2}+\|u\|_{H^{\delta}}^{2}<\infty
$$

Therefore $A_{2} u \in H^{\delta}\left(\mathrm{R}^{n}\right)$. Using the definition of $A_{2}$ and Lemma 2.2 we conclude $u \in H^{4-\delta}\left(\mathrm{R}^{n}\right)$. Now, due to $v=u-f \in H^{2}\left(\mathrm{R}^{n}\right)$ it follows that $v+$ $A_{2} u=g$ is true. We conclude that $B_{1}$ is maximal. But $H^{A-\delta}\left(R^{n}\right) \times H^{2}\left(R^{n}\right)$ is dense in the energy space $X$. Then by by Lumer-Phillips theorem, we obtain that $B_{1}$ is infinitesimal generator of a contraction semigroup of class $C_{0}$ in $X$.

Lemma 2.5: The operator $J_{1}: X \rightarrow X$ is a bounded linear operator.

Proof: The fact that $J_{1}$ is linear is obvious. The prove that $J_{1}$ is bounded on $X$ follows from the estimate

$$
\begin{aligned}
& \left\|J_{1}(U)\right\|_{H^{2} \times H^{\delta}}=\left\|\left(I+(-\Delta)^{\delta}\right)^{-1}\left(u-(-\Delta)^{\theta} v\right)\right\|_{H^{\delta}}=\int_{\mathbb{R}^{n}}\left(1+|\xi|^{2 \delta}\right)\left|\frac{\hat{u}-|\xi|^{2 \theta} \hat{v}}{1+|\xi|^{2 \delta}}\right|^{2} d \xi \\
& \leq 2 \int_{\mathbb{R}^{n}}|\hat{u}|^{2} d \xi+2 \int_{\mathbb{R}^{n}} \frac{|\xi|^{4 \theta}}{1+|\xi|^{2 \delta}}|\hat{v}|^{2} d \xi \leq 2\|u\|_{H^{2}}+2\|v\|_{H^{\delta}} \leq 2\|U\|_{H^{2} \times H^{\delta}},
\end{aligned}
$$

which holds because

$$
\frac{|\xi|^{4 \theta}}{1+|\xi|^{2 \delta}} \leq 1+|\xi|^{2 \delta}
$$

when $0 \leq \theta<\delta$.

The fact that $B_{1}$ is infinitesimal generator of a contraction semigroup of class $C_{0}$ in $X$ and $J_{1}$ is a bounded linear operator on $X$ we conclude by theorem 1.3 that $B_{1}+J_{1}$ is infinitesimal generator of a semigroup of class $C_{0}$. Let $S_{1}:[0, \infty) \rightarrow \mathrm{L}(X)$ be the semigroup of class $C_{0}$ in $X$ generated by $B_{1}+J_{1}$ then $U(t)=S_{1}(t) U_{0}$ is the solution of the Cauchy Problem (18).

For initial data $U_{0}=\left(u_{0}, u_{1}\right) \in X$ then the first component $u=u(t)$ of $U(t)=\left(u, u_{t}\right)$ is the unique weak solution of the system (4) in the class. 


$$
u \in C\left([0, \infty), H^{2}\left(\mathbb{R}^{n}\right)\right) \cap C^{1}\left([0, \infty), H^{\delta}\left(\mathbb{R}^{n}\right)\right)
$$

If the initial data $U_{0}=\left(u_{0}, u_{1}\right) \in D\left(B_{1}\right)=H^{A-\delta}\left(\mathrm{R}^{n}\right) H^{2}\left(\mathrm{R}^{n}\right)$ then $u=u(t)$ is the unique strong solution of the system (4) and satisfies

$$
u \in C\left([0, \infty), H^{4-\delta}\left(\mathbb{R}^{n}\right)\right) \cap C^{1}\left([0, \infty), H^{2}\left(\mathbb{R}^{n}\right)\right) \cap C^{2}\left([0, \infty), H^{\delta}\left(\mathbb{R}^{n}\right)\right) .
$$

\subsection{Case $0 \leq \delta \leq \theta$ and $0 \leq \theta \leq 2$}

We first observe the conditions on fractional powers, $0 \leq \delta \leq \theta$ and 0 $\leq \theta \leq 2$, implies $0 \leq \delta \leq 2$ and because that $H^{2}\left(\mathrm{R}^{n}\right) \subset H^{\delta}\left(\mathrm{R}^{n}\right)$. So, for the case in consideration. we can also consider again the energy space as $X=$ $H^{2}\left(\mathrm{R}^{n}\right) \times H^{\delta}\left(\mathrm{R}^{n}\right)$.

Similarly to the previous section, we can consider operators $B_{2}$ and $J_{2}$. For $v=u_{t}$ we have

$$
v_{t}=u_{t t}=-\left(I+(-\Delta)^{\delta}\right)^{-1}\left(\alpha \Delta^{2}-\Delta\right) u-\left(I+(-\Delta)^{\delta}\right)^{-1}(-\Delta)^{\theta} v
$$

Now, considering the operators $A_{2}$ and $A_{\theta}$, we can rewrite the Cauchy Problem (4) in matrix form as follows

$$
\left\{\begin{array}{l}
\frac{d U}{d t}=B_{2} U+J_{2}(U) \\
U(0)=U_{0}
\end{array}\right.
$$

where $U=U(t)=(u, v), U_{0}=\left(u_{0}, u_{1}\right)$, and the operators $B_{2}$ : $H^{4-\delta}\left(\mathrm{R}^{n}\right) \times H^{2}\left(\mathrm{R}^{n}\right) \rightarrow X$ and $J_{2}: X \rightarrow X$ are given by

$$
B_{2}=\left(\begin{array}{ll}
0 & I \\
-A_{2} & -A_{\theta}
\end{array}\right) \quad \text { and } \quad J_{2}(U)=\left(\begin{array}{l}
0 \\
\left(I+(-\Delta)^{\delta}\right)^{-1}(u+v)
\end{array}\right) .
$$

Similarly to the previous subsection, we may prove that $B_{2}$ is infinitesimal generator of a contraction semigroup of class $C_{0}$ in $X$ and $J_{2}$ is linear and bounded on $X$. Then, by Theorem 1.3 we conclude $B_{2}+J_{2}$ is 
infinitesimal generator of a semigroup of class $C_{0}$. Let $S_{2}:[0, \infty) \rightarrow L(X)$ the semigroup generated by $B_{2}+J_{2}$. Then $U(t)=S_{2}(t) U_{0}$ is the unique solution of the Cauchy Problem (18) for the case on $\delta$ and $\theta$ in this subsection.

Then, for initial data $U_{0}=\left(u_{0}, u_{1}\right) \in X$ the first component $u(t)$ of $U(t)$ $=S_{2}(t) U_{0} \in C[0, \infty), X$ is the unique weak solution of the linear problem (4) and satisfies

$$
u \in C\left([0, \infty), H^{2}\left(\mathbb{R}^{n}\right)\right) \cap C^{1}\left([0, \infty), H^{\delta}\left(\mathbb{R}^{n}\right)\right) .
$$

If the initial data $U_{0}=\left(u_{0}, u_{1}\right) \in D\left(B_{2}\right)=H^{A-\delta}\left(\mathrm{R}^{n}\right) H^{2}\left(\mathrm{R}^{n}\right)$ then $u(t)$ is the unique strong solution of (4) in the class

$$
u \in C\left([0, \infty), H^{4-\delta}\left(\mathbb{R}^{n}\right)\right) \cap C^{1}\left([0, \infty), H^{2}\left(\mathbb{R}^{n}\right)\right) \cap C^{2}\left([0, \infty), H^{\delta}\left(\mathbb{R}^{n}\right)\right) .
$$

\section{DECAY RATES: LINEAR PROBLEM}

The following theorems are proved in Charão-Horbach-Ikehata. These results show almost optimal decay rates to the norm of energy and $L^{2}-$ norm of the solution to the linear Cauchy problem (4).

Theorem 3.1: Let $0 \leq \theta<\delta$. Then, the following decay rates are valid for the energy norm of the solution $u(t, x)$ of (4).

i) Let $n \geq 1$ and $0 \leq \theta \leq \frac{1}{2}$. Then, for initial data $u_{0} \in H^{\frac{(\delta-\theta) n}{2-2 \theta}+2}\left(\mathbb{R}^{n}\right) \cap L^{1}\left(\mathbb{R}^{n}\right)$ and $u_{1} \in H^{\frac{(\delta-\theta) n}{2-2 \theta}+\delta}\left(\mathbb{R}^{n}\right) \cap L^{1}\left(\mathbb{R}^{n}\right)$, it holds that

$$
\begin{aligned}
& \int_{\mathbb{R}^{n}}\left(\left|u_{t}\right|^{2}+\left|(-\Delta)^{\delta} u_{t}\right|^{2}+\alpha|\Delta u|^{2}+\left|(-\Delta)^{1 / 2} u\right|^{2}\right) d x \\
& \leq C t^{-\frac{n}{2-2 \theta}}\left(\left\|u_{0}\right\|_{L^{1}}^{2}+\left\|u_{0}\right\|_{H^{\frac{(\delta-\theta) n}{2-2 \theta}+2}}^{2}+\left\|u_{1}\right\|_{L^{1}}^{2}+\left\|u_{1}\right\|_{H^{\frac{(\delta-\theta) n}{2-2 \theta}+\delta}}^{2}\right) .
\end{aligned}
$$


ii) Let $n \geq 1$ and $\frac{1}{2}<\theta \leq \frac{2+\delta}{2}$. Then, for initial data $u_{0} \in H^{\frac{(\delta-\theta) n}{2 \theta}+2}\left(\mathbb{R}^{n}\right) \cap L^{1}\left(\mathbb{R}^{n}\right)$ and $u_{1} \in H^{\frac{(\delta-\theta) n}{2 \theta}+\delta}\left(\mathbb{R}^{n}\right) \cap L^{1}\left(\mathbb{R}^{n}\right)$, then

$$
\begin{aligned}
& \int_{\mathbb{R}^{n}}\left(\left|u_{t}\right|^{2}+\left|(-\Delta)^{\delta} u_{t}\right|^{2}+\alpha|\Delta u|^{2}+\left|(-\Delta)^{1 / 2} u\right|^{2}\right) d x \\
& \leq C t^{-\frac{n}{2 \theta}}\left(\left\|u_{0}\right\|_{L^{1}}^{2}+\left\|u_{0}\right\|_{H^{\frac{(\delta-\theta) n}{2 \theta}}+2}^{2}+\left\|u_{1}\right\|_{L^{1}}^{2}+\left\|u_{1}\right\|_{H^{\frac{(\delta-\theta) n}{2 \theta}}+\delta}^{2}\right) .
\end{aligned}
$$

Theorem 3.2: Let $0 \leq \theta<\delta$. Then, the following decay rates are valid for the $L^{2}$-norm of solution $u(t, x)$ of Problem (4).

i) Let $0 \leq \theta \leq \frac{1}{2}$ and $n \geq 1$. Then, for initial data $u_{0} \in L^{1}\left(\mathbb{R}^{n}\right) \cap H^{\frac{(\delta-\theta) n}{2-2 \theta}}\left(\mathbb{R}^{n}\right)$ and $u_{1} \in \dot{W}^{-1,1}\left(\mathbb{R}^{n}\right) \cap H^{\frac{(\delta-\theta) n}{2-2 \theta}+\delta-2}\left(\mathbb{R}^{n}\right)$, then

$$
\int_{\mathbb{R}^{n}}|u|^{2} d x \leq C t^{-\frac{n}{2-2 \theta}}\left(\left\|u_{1}\right\|_{\dot{W}^{-1,1}}^{2}+\left\|u_{1}\right\|_{H^{\frac{(\delta-\theta) n}{2-2 \theta}+\delta-2}}^{2}+\left\|u_{0}\right\|_{L^{1}}^{2}+\left\|u_{0}\right\|_{H^{\frac{(\delta-\theta) n}{2-2 \theta}}}^{2}\right) .
$$

In addition, if $n \geq 3$ then, for initial data $u_{0} \in L^{1}\left(\mathbb{R}^{n}\right) \cap H^{\frac{(\delta-\theta) n}{2-2 \theta}}\left(\mathbb{R}^{n}\right)$ and $u_{1} \in$ $L^{1}\left(\mathbb{R}^{n}\right) \cap H^{\frac{(\delta-\theta)(n-4 \theta)}{2-2 \theta}+\delta-2}\left(\mathbb{R}^{n}\right)$, it holds that, for a fixed $\tau>0$,

$$
\int_{\mathbb{R}^{n}}|u|^{2} d x \leq C t^{-\frac{n-4 \theta}{2-2 \theta}+\tau}\left(\left\|u_{1}\right\|_{L^{1}}^{2}+\left\|u_{0}\right\|_{L^{1}}^{2}\right)+C t^{-\frac{n-4 \theta}{2-2 \theta}}\left\|u_{1}\right\|_{H^{\frac{(\delta-\theta)(n-4 \theta)}{2-2 \theta}+\delta-2}}^{2}+C t^{-\frac{n}{2-2 \theta}}\left\|u_{0}\right\|_{H^{\frac{(\delta-\theta) n}{2-2 \theta}}}^{2} .
$$

ii) Let $\frac{1}{2}<\theta \leq \frac{2+\delta}{2}$ and $n \geq 1$. Then, for initial data $u_{0} \in L^{1}\left(\mathbb{R}^{n}\right) \cap H^{\frac{(\delta-\theta) n}{2 \theta}}\left(\mathbb{R}^{n}\right)$ and $u_{1} \in \dot{W}^{-1,1}\left(\mathbb{R}^{n}\right) \cap H^{\frac{(\delta-\theta) n}{2 \theta}+\delta-2}\left(\mathbb{R}^{n}\right)$, it holds

$$
\int_{\mathbb{R}^{n}}|u|^{2} d x \leq C t^{-\frac{n}{2 \theta}}\left(\left\|u_{1}\right\|_{W^{-1,1}}^{2}+\left\|u_{1}\right\|_{H^{\frac{(\delta-\theta) n}{2 \theta}+\delta-2}}^{2}+\left\|u_{0}\right\|_{L^{1}}^{2}+\left\|u_{0}\right\|_{H^{\frac{(\delta-\theta) n}{2 \theta}}}^{2}\right) .
$$

In addition, if $n \geq 3, u_{0} \in L^{1}\left(\mathbb{R}^{n}\right) \cap H^{\frac{(\delta-\theta) n}{2 \theta}}\left(\mathbb{R}^{n}\right)$ and $u_{1} \in L^{1}\left(\mathbb{R}^{n}\right) \cap H^{\frac{(\delta-\theta)(n-2)}{2 \theta}+\delta-2}\left(\mathbb{R}^{n}\right)$, then

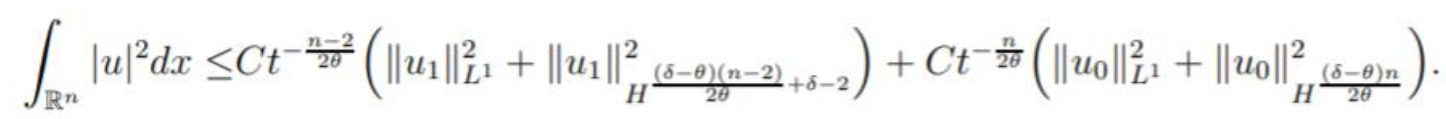

Theorem 3.3: Let $0 \leq \delta \leq \theta$ and $u_{0} \in H^{2}\left(\mathrm{R}^{n}\right) \cap L^{1}(\mathrm{R} n)$ and $u_{1} \in H^{\bar{P}}\left(\mathrm{R}^{n}\right)$ $\cap L^{1}\left(R^{n}\right)$. Then, the following decay rates are valid for the energy norm of the solution $u(t, x)$ of $(4)$. 
i) Let $n \geq 1$ and $0 \leq \theta \leq \frac{1}{2}$. Then

$$
\begin{aligned}
& \int_{\mathbb{R}^{n}}\left(\left|u_{t}\right|^{2}+\left|(-\Delta)^{\delta} u_{t}\right|^{2}+\alpha|\Delta u|^{2}+\left|(-\Delta)^{1 / 2} u\right|^{2}\right) d x \\
& \leq C t^{-\frac{n}{2-2 \theta}}\left(\left\|u_{0}\right\|_{L^{1}}^{2}+\left\|u_{1}\right\|_{L^{1}}^{2}\right)+e^{-\frac{\varepsilon}{10} t}\left(\left\|u_{0}\right\|_{H^{2}}^{2}+\left\|u_{1}\right\|_{H^{\delta}}^{2}\right) .
\end{aligned}
$$

ii) Let $n \geq 1$ and $\frac{1}{2}<\theta \leq \frac{2+\delta}{2}$. Then

$$
\begin{aligned}
& \int_{\mathbb{R}^{n}}\left(\left|u_{t}\right|^{2}+\left|(-\Delta)^{\delta} u_{t}\right|^{2}+\alpha|\Delta u|^{2}+\left|(-\Delta)^{1 / 2} u\right|^{2}\right) d x \\
& \leq C t^{-\frac{n}{2 \theta}}\left(\left\|u_{0}\right\|_{L^{1}}^{2}+\left\|u_{1}\right\|_{L^{1}}^{2}\right)+e^{-\frac{\varepsilon}{10} t}\left(\left\|u_{0}\right\|_{H^{2}}^{2}+\left\|u_{1}\right\|_{H^{\delta}}^{2}\right) .
\end{aligned}
$$

Theorem 3.4: Let $0 \leq \delta \leq \theta$. Then the following decay rates are valid for the $L^{2}$-norm of the solution $u(t, x)$ of Problem (4).

i) Let $0 \leq \theta \leq \frac{1}{2}$ and $n \geq 1$. Then, for initial data $u_{0} \in H^{2}\left(\mathbb{R}^{n}\right) \cap L^{1}\left(\mathbb{R}^{n}\right)$ and $u_{1} \in H^{\delta}\left(\mathbb{R}^{n}\right) \cap W^{-1,1}\left(\mathbb{R}^{n}\right)$

$$
\int_{\mathbb{R}^{n}}|u|^{2} d x \leq C t^{-\frac{n}{2-2 \theta}}\left(\left\|u_{1}\right\|_{W^{-1,1}}^{2}+\left\|u_{0}\right\|_{L^{1}}^{2}\right)+e^{-\frac{\varepsilon}{10} t}\left(\left\|u_{0}\right\|_{H^{2}}^{2}+\left\|u_{1}\right\|_{H^{\delta}}^{2}\right) .
$$

In addition, if $n \geq 3$ then, for initial data $u_{0} \in H^{2}\left(\mathbb{R}^{n}\right) \cap L^{1}\left(\mathbb{R}^{n}\right)$ and $u_{1} \in H^{\delta}\left(\mathbb{R}^{n}\right) \cap$ $L^{1}\left(\mathbb{R}^{n}\right)$ and a fixed $\tau>0$,

$$
\int_{\mathbb{R}^{n}}|u|^{2} d x \leq C t^{-\frac{n-4 \theta}{2-2 \theta}+\tau}\left(\left\|u_{1}\right\|_{L^{1}}^{2}+\left\|u_{0}\right\|_{L^{1}}^{2}\right)+e^{-\frac{\varepsilon}{10} t}\left(\left\|u_{0}\right\|_{H^{2}}^{2}+\left\|u_{1}\right\|_{H^{\delta}}^{2}\right) .
$$

ii) Let $\frac{1}{2}<\theta \leq \frac{2+\delta}{2}$ and $n \geq 1$. Then, for initial data $u_{0} \in H^{2}\left(\mathbb{R}^{n}\right) \cap L^{1}\left(\mathbb{R}^{n}\right)$ and $u_{1} \in H^{\delta}\left(\mathbb{R}^{n}\right) \cap \dot{W}^{-1,1}\left(\mathbb{R}^{n}\right)$,

$$
\int_{\mathbb{R}^{n}}|u|^{2} d x \leq C t^{-\frac{n}{2 \theta}}\left(\left\|u_{1}\right\|_{\dot{W}^{-1,1}}^{2}+\left\|u_{0}\right\|_{L^{1}}^{2}\right)+e^{-\frac{\varepsilon}{10} t}\left(\left\|u_{0}\right\|_{H^{2}}^{2}+\left\|u_{1}\right\|_{H^{\delta}}^{2}\right) .
$$

In addition, if $n \geq 3$ then, for initial data $u_{0} \in H^{2}\left(\mathbb{R}^{n}\right) \cap L^{1}\left(\mathbb{R}^{n}\right)$ and $u_{1} \in H^{\delta}\left(\mathbb{R}^{n}\right) \cap$ $L^{1}\left(\mathbb{R}^{n}\right)$, it holds

$$
\int_{\mathbb{R}^{n}}|u|^{2} d x \leq C t^{-\frac{n-2}{2 \theta}}\left\|u_{1}\right\|_{L^{1}}^{2}+C t^{-\frac{n}{2 \theta}}\left\|u_{0}\right\|_{L^{1}}^{2}+e^{-\frac{\varepsilon}{10} t}\left(\left\|u_{0}\right\|_{H^{2}}^{2}+\left\|u_{1}\right\|_{H^{\delta}}^{2}\right) .
$$




\section{EXISTENCE AND UNIQUENESS: SEMILINEAR PROBLEM}

In this section we consider the Cauchy problem associated to a semilinear equation in $\mathrm{R}^{n}$ of Boussineq/plate type with fractional damping given by

$$
\left\{\begin{array}{l}
u_{t t}+(-\Delta)^{\delta} u_{t t}+\alpha \Delta^{2} u-\Delta u+(-\Delta)^{\theta} u_{t}=\beta(-\Delta)^{\gamma}\left(u^{p}\right) \\
u(0, x)=u_{0}(x) \\
u_{t}(0, x)=u_{1}(x)
\end{array}\right.
$$

where $u=u(t, x),(t, x) \in(0, \infty) \times \mathrm{R}^{n}, a>0, \beta /=0$ and $p>1$ integer. The fractional powers of the Laplacian operator are considered as follows $0 \leq \delta \leq 2,0 \leq \theta \leq \frac{2+\delta}{2}$ and $\frac{1}{2} \leq \gamma \leq \frac{2+\delta}{2}$.

In case $\delta=2, y=1, p=2$ and $\theta=1$ we have a sixth order Boussinesq equation under the effects of a hydrodynamic dissipation (see WAANG, S. e XUE, H., 2008; DARIPA, P. e HUA, W., 2012). If $\theta=0, \delta=1$, $y=1 / 2, \beta=0$ and $n=2$ we have a semilinear plate equation under the effects of a frictional dissipation (see CHARÃO, R. C., DA LUZ, C. R. e IKEHATA, R, 2013; DA LUZ, C. R. e CHARAO, R. C, 2009; and SUGITANI, Y. e KAWASHIMA, S, 2013).

Similar to the linear case, to study the existence of solutions we need to consider two cases.
i) $0 \leq \theta<\delta$ and $0 \leq \delta \leq 2$
ii) $0 \leq \delta \leq \theta$ and $0 \leq \theta \leq \frac{2+\delta}{2}$.

We reduce the order of the Cauchy Problem (19) and rewrite it in the following matrix form

$$
\left\{\begin{array}{l}
\frac{d U}{d t}=B U+F(U) \\
U(0)=U_{0}
\end{array}\right.
$$

where $U=\left(u, u_{t}\right), U_{0}=\left(u_{0}, u_{1}\right)$ and the operator $B$ is define in the Section 3 according to the both cases above mentioned. So, in both cases $B$ is the infinitesimal generator of a contraction semigroup of $C_{0^{-}}$ 
class in $X$. The operator $F$ is the operator that contains the non-linear term.

\subsection{Local Existence}

To prove the local existence, since $B$ generate a semigroup, we need only to show that the operator $F$ is well defined as an operator $F: D(B) \rightarrow$ $D(B)$ and it is Lipschitz continuous on bounded sets of $D(B)$. After prove this property of $F$ we can take $U_{0} \in D(B)$ and using Theorem 1.4 one can conclude that there exist a unique solution $U=U(t)$ in a maximal interval $\left[0, T_{m}\right)$ such that one and only one of the following conditions is true.
(i) $T_{m}=\infty$
(ii) $T_{m}<\infty$ and $\lim _{t \rightarrow T_{m}}\|U\|_{X}+\|B U\|_{X}=\infty$.

In addition, we have $U \in C^{1}\left[0, T_{m}\right), X \cap C\left[0, T_{m}\right), D(B)$.

\subsubsection{Case $0 \leq \theta<\delta$ and $0 \leq \delta \leq 2$}

To show the local existence for this case, we should consider the fractional power $r$ in the interval $0 \leq \gamma \leq \frac{2+\delta}{2}$. As in the linear case we consider the usual energy space $X$ and we rewrite the system (19) in the matrix form

$$
\left\{\begin{array}{l}
\frac{d U}{d t}=B_{1} U+F_{1}(U) \\
U(0)=U_{0}
\end{array}\right.
$$

where $U=(u, v) \in X, U_{0}=\left(u_{0}, u_{1}\right) \in X$ and the operators $B_{1}$ : $H^{A-\delta}\left(\mathrm{R}^{n}\right) \times H^{2}\left(\mathrm{R}^{n}\right) \rightarrow X$ and $F_{1}: D\left(B_{1}\right) \rightarrow D\left(B_{1}\right)$ are given by

$$
B_{1}=\left(\begin{array}{ll}
0 & I \\
-A_{2} & 0
\end{array}\right) \quad \text { and } \quad F_{1}(U)=\left(\begin{array}{l}
0 \\
\left(I+(-\Delta)^{\delta}\right)^{-1}\left(u-(-\Delta)^{\theta} v+\beta(-\Delta)^{\gamma} u^{p}\right)
\end{array}\right) .
$$

Lemma 4.1: The operator $F_{1}: D\left(B_{1}\right) \rightarrow D\left(B_{1}\right)$ is well define for $0 \leq \gamma$ $\leq \frac{2+\delta}{2}$ and $n<8-2 \delta$. 
Proof: We consider $U=(u, v) \in D\left(B_{1}\right)$ and we show that $F_{1}(u, v) \in$ $D\left(B_{1}\right)$. By definition of $F_{1}$ and norm in $H^{R}$ we have

$$
\begin{aligned}
& \left\|F_{1}(u, v)\right\|_{H^{4-\delta} \times H^{2}}^{2}=\left\|\left(I+(-\Delta)^{\delta}\right)^{-1}\left(u-(-\Delta)^{\theta} v+\beta(-\Delta)^{\gamma} u^{p}\right)\right\|_{H^{2}}^{2} \\
& =\left.\int_{\mathbb{R}^{n}} \frac{\left(1+|\xi|^{2}+\alpha|\xi|^{4}\right)}{\left(1+|\xi|^{2 \delta}\right)^{2}}|\hat{u}-| \xi\right|^{2 \theta} \hat{v}+\left.\beta|\xi|^{2 \gamma} \widehat{u^{p}}\right|^{2} d \xi \\
& \leq C \int_{\mathbb{R}^{n}} \frac{\left(1+|\xi|^{2}+\alpha|\xi|^{4}\right)}{\left(1+|\xi|^{2 \delta}\right)^{2}}\left(|\hat{u}|^{2}+|\xi|^{4 \theta}|\hat{v}|^{2}+|\xi|^{4 \gamma}\left|\widehat{u^{p}}\right|^{2}\right) d \xi \\
& \leq C \int_{\mathbb{R}^{n}}\left[\left(1+|\xi|^{2(2-2 \delta)}\right)|\hat{u}|^{2}+\left(1+|\xi|^{2(2+2 \theta-2 \delta)}\right)|\hat{v}|^{2}+\left(1+|\xi|^{2(2+2 \gamma-2 \delta)}\right)\left|\widehat{u^{p}}\right|^{2}\right] d \xi \\
& \leq C\|u\|_{H^{2-2 \delta}}^{2}+C\|v\|_{H^{2+2 \theta-2 \delta}}^{2}+C\left\|u^{p}\right\|_{H^{2+2 \gamma-2 \delta}}^{2},
\end{aligned}
$$

due to the assumptions $2-2 \delta \leq 4-\delta, 2+2 \theta-2 \delta<2$ and $2+2 \gamma$ $-2 \delta \leq 4-\delta$.

Thus, from the definition of norm in $H^{s}\left(\mathrm{R}^{n}\right)$ and the natural embedding of $H_{s}\left(\mathrm{R}^{n}\right)$ in $\operatorname{Hr}\left(\mathrm{R}^{n}\right)$ for $s \geq r$, we get the following estimate

$$
\left\|F_{1}(u, v)\right\|_{H^{4-\delta} \times H^{2}}^{2} \leq C\|u\|_{H^{4-\delta}}^{2}+C\|v\|_{H^{2}}^{2}+C\left\|u^{p}\right\|_{H^{4-\delta}}^{2} .
$$

Now using Lemma 1.3 with $s=4-\delta$, we obtain for $n<8-2 \delta$

$$
\left\|F_{1}(u, v)\right\|_{H^{4-\delta} \times H^{2}}^{2} \leq C\|u\|_{H^{4-\delta}}^{2}+C\|v\|_{H^{2}}^{2}+C\|u\|_{H^{4-\delta}}^{2 p}<+\infty .
$$

Lemma 4.2: Let $1 \leq n<8-2 \delta, 0 \leq \theta<\delta, 0 \leq \delta \leq 2,0 \leq \mathrm{Y} \leq \frac{2+\delta}{2}$ and $p$ $>1$ integer. let $U=(u, v)$ and $W=(w, z)$ such that $U, W \in D\left(B_{1}\right)=H^{4-\delta}$ $\left(\mathrm{R}^{\mathrm{n}}\right) \times H^{2}\left(\mathrm{R}^{\mathrm{n}}\right)$. Then

$$
\left\|F_{1}(U)-F_{1}(W)\right\|_{X} \leq C\left(1+\left\|B_{1}(U)\right\|_{X}^{p-1}+\left\|B_{1}(W)\right\|_{X}^{p-1}\right)\left\|B_{1}(U-W)\right\|_{X} .
$$

Proof: For $U=(u, v)$ and $W=(w, z)$ in $H^{A-\delta}\left(\mathrm{R}^{n}\right) \times H^{2}\left(\mathrm{R}^{n}\right)$ we have 


$$
\begin{aligned}
& \left\|F_{1}(U)-F_{1}(W)\right\|_{X}^{2} \\
& \leq\left\|\left(I+(-\Delta)^{\delta}\right)^{-1}\left((u-w)-(-\Delta)^{\theta}(v-z)\right)\right\|_{H^{\delta}}^{2}+\left\|\beta\left(I+(-\Delta)^{\delta}\right)^{-1}(-\Delta)^{\gamma}\left(u^{p}-w^{p}\right)\right\|_{H^{\delta}}^{2} \\
& =\left.\left.\int_{\mathbb{R}^{n}} \frac{1}{\left(1+|\xi|^{2 \delta}\right)}|(\hat{u}-\hat{w})-| \xi\right|^{2 \theta}(\hat{v}-\hat{z})\right|^{2} d \xi+\left.\left.\int_{\mathbb{R}^{n}} \frac{1}{\left(1+|\xi|^{2 \delta}\right)}|\beta| \xi\right|^{2 \gamma}\left(\widehat{u^{p}}-\widehat{w^{p}}\right)\right|^{2} d \xi \\
& \leq C \int_{\mathbb{R}^{n}}|\hat{u}-\hat{w}|^{2}+\left(1+|\xi|^{2(2 \theta-\delta)}\right)|\hat{v}-\hat{z}|^{2} d \xi+C \int_{\mathbb{R}^{n}}\left(1+|\xi|^{2(2 \gamma-\delta)}\right)\left|\widehat{u^{p}}-\widehat{w^{p}}\right|^{2} d \xi \\
& \leq C\|u-w\|_{H^{4-\delta}}^{2}+C\|v-z\|_{H^{2}}^{2}+C\left\|u^{p}-w^{p}\right\|_{H^{4-\delta}}^{2},
\end{aligned}
$$

because $2 \theta-\delta<2$ and $2 \gamma-\delta \leq 4-\delta$.

Now, using Lemma 1.5 with $s=4-\delta$, for $n<8-2 \delta$ we have

$$
\left\|F_{1}(U)-F_{1}(W)\right\|_{X}^{2} \leq C\|u-w\|_{H^{4-\delta}}^{2}+C\|v-z\|_{H^{2}}^{2}+C\left(\|u\|_{H^{4-\delta}}^{p-1}+\|w\|_{H^{4-\delta}}^{p-1}\right)^{2}\|u-w\|_{H^{4-\delta}}^{2} .
$$

The estimate (see lemma 2.2) $\|u\|_{H^{4-\delta} \leq C} \leq C A_{2} u \|_{H \delta}$ for all $u \in D\left(A_{2}\right)$ and the defini- tion of operator $B_{1}$ imply that

$$
\begin{aligned}
& \left\|F_{1}(U)-F_{1}(W)\right\|_{X}^{2} \\
& \leq C\left\|A_{2}(u-w)\right\|_{H^{\delta}}^{2}+C\|v-z\|_{H^{2}}^{2}+C\left(\left\|A_{2} u\right\|_{H^{\delta}}^{p-1}+\left\|A_{2} w\right\|_{H^{\delta}}^{p-1}\right)^{2}\left\|A_{2}(u-w)\right\|_{H^{\delta}}^{2} \\
& \leq C\left\|B_{1}(U-W)\right\|_{X}^{2}+C\left(\left\|B_{1} U\right\|_{X}^{p-1}+\left\|B_{1} W\right\|_{X}^{p-1}\right)^{2}\left\|B_{1}(U-W)\right\|_{X}^{2} \\
& \leq C\left(1+\left\|B_{1} U\right\|_{X}^{p-1}+\left\|B_{1} W\right\|_{X}^{p-1}\right)^{2}\left\|B_{1}(U-W)\right\|_{X}^{2} .
\end{aligned}
$$

Lemma 4.3: Let $1 \leq n<8-2 \delta, 0 \leq \theta<\delta, 0 \leq \delta \leq 2,0 \leq \gamma \leq \frac{2+\delta}{2}$ and $p>$ 1 integer. Let $U=(u, v)$ and $W=(w, z)$ such that $U, W \in D\left(B_{1}\right)=H^{4-\delta}\left(\mathbf{R}^{n}\right) \times$ $H^{2}\left(\mathrm{R}^{n}\right)$. Then there exist a constant $\mathrm{C}>0$ such that

$$
\left\|B_{1}\left(F_{1}(U)-F_{1}(W)\right)\right\|_{X} \leq C\left(1+\left\|B_{1}(U)\right\|_{X}^{p-1}+\left\|B_{1}(W)\right\|_{X}^{p-1}\right)\left\|B_{1}(U-W)\right\|_{X} .
$$

Proof: For $U=(u, v)$ and $W=(w, z)$ in $H^{4-\delta}\left(\mathrm{R}^{n}\right) \times H^{2}\left(\mathrm{R}^{n}\right)$ we have 


$$
\begin{aligned}
& \left\|B_{1}\left(F_{1}(U)-F_{1}(W)\right)\right\|_{X}^{2} \\
& \leq\left\|\left(I+(-\Delta)^{\delta}\right)^{-1}\left|(u-w)-(-\Delta)^{\theta}(v-z)\right|\right\|_{H^{2}}^{2}+\left\|\beta\left(I+(-\Delta)^{\delta}\right)^{-1}(-\Delta)^{\gamma}\left(u^{p}-w^{p}\right)\right\|_{H^{2}}^{2} \\
& \leq\left.\left.\int_{\mathbb{R}^{n}} \frac{\left(1+|\xi|^{2}+\alpha|\xi|^{4}\right)}{\left(1+|\xi|^{2 \delta}\right)^{2}}|(\hat{u}-\hat{w})-| \xi\right|^{2 \theta}(\hat{v}-\hat{z})\right|^{2} d \xi+\left.\left.\int_{\mathbb{R}^{n}} \frac{\left(1+|\xi|^{2}+\alpha|\xi|^{4}\right)}{\left(1+|\xi|^{2 \delta}\right)^{2}}|\beta| \xi\right|^{2 \gamma}\left(\widehat{u^{p}}-\widehat{w^{p}}\right)\right|^{2} d \xi \\
& \leq C \int_{\mathbb{R}^{n}}\left(1+|\xi|^{2(2-2 \delta)}\right)|\hat{u}-\hat{w}|^{2}+\left(1+|\xi|^{2(2+2 \theta-2 \delta)}\right)|\hat{v}-\hat{z}|^{2} d \xi+C \int_{\mathbb{R}^{n}}\left(1+|\xi|^{2(2+2 \gamma-2 \delta)}\right)\left|\widehat{u^{p}}-\widehat{w^{p}}\right|^{2} d \xi \\
& =C\|u-w\|_{H^{2-2 \delta}}^{2}+C\|v-z\|_{H^{2+2 \theta-2 \delta}}^{2}+C\left\|u^{p}-w^{p}\right\|_{H^{2+2 \gamma-2 \delta}}^{2},
\end{aligned}
$$

since we have assumed $2-2 \delta \leq 4-\delta, 2+2 \theta-2 \delta<2$ and $2+2 \gamma$ $-2 \delta \leq 4-\delta$.

The above estimate combined with lemma 1.5 imply for $n<8-2 \delta$ the estimate of lemma.

$$
\begin{aligned}
& \left\|B_{1}\left(F_{1}(U)-F_{1}(W)\right)\right\|_{X}^{2} \leq C\|u-w\|_{H^{4-\delta}}^{2}+C\|v-z\|_{H^{2}}^{2}+C\left\|u^{p}-w^{p}\right\|_{H^{4-\delta}}^{2} \\
& \leq C\|u-w\|_{H^{4-\delta}}^{2}+C\|v-z\|_{H^{2}}^{2}+C\left(\|u\|_{H^{4-\delta}}^{p-1}+\|w\|_{H^{4-\delta}}^{p-1}\right)^{2}\|u-w\|_{H^{4-\delta}}^{2} \\
& \leq C\left\|A_{2}(u-w)\right\|_{H^{\delta}}^{2}+C\|v-z\|_{H^{2}}^{2}+C\left(\left\|A_{2} u\right\|_{H^{\delta}}^{p-1}+\left\|A_{2} w\right\|_{H^{\delta}}^{p-1}\right)^{2}\left\|A_{2}(u-w)\right\|_{H^{\delta}}^{2} \\
& \leq C\left\|B_{1}(U-W)\right\|_{X}^{2}+C\left(\left\|B_{1} U\right\|_{X}^{p-1}+\left\|B_{1} W\right\|_{X}^{p-1}\right)^{2}\left\|B_{1}(U-W)\right\|_{X}^{2} \\
& \leq C\left(1+\left\|B_{1} U\right\|_{X}^{p-1}+\left\|B_{1} W\right\|_{X}^{p-1}\right)^{2}\left\|B_{1}(U-W)\right\|_{X}^{2}
\end{aligned}
$$

Finally, combining the Lemmas 4.2 and 4.3 we conclude that

$$
\left\|F_{1}(U)-F_{1}(W)\right\|_{X}+\left\|B_{1}\left(F_{1}(U)-F_{1}(W)\right)\right\|_{X} \leq C\left(1+\left\|B_{1}(U)\right\|_{X}^{p-1}+\left\|B_{1}(W)\right\|_{X}^{p-1}\right)\left\|B_{1}(U-W)\right\|_{X}
$$

Therefore, given a constant $M>0$ and considering $U, W \in H^{4-\delta}\left(\mathrm{R}^{n}\right)$ $H^{2}\left(R^{n}\right)$ such that

$$
\left\|B_{1}(U)\right\|_{X} \leq M \quad \text { and } \quad\left\|B_{1}(W)\right\|_{X} \leq M
$$

we have, for $L_{M}=1+2 M^{p-1}$, the following estimate

$$
\left\|F_{1}(U)-F_{1}(W)\right\|_{X}+\left\|B_{1}\left(F_{1}(U)-F_{1}(W)\right)\right\|_{X} \leq C L_{M}\left\|B_{1}(U-W)\right\|_{X} .
$$


Thus, we conclude that $F_{1}$ is Lipschitz continuous on bounded sets of $D\left(B_{1}\right)$. Then, the fact that $B_{1}$ is infinitesimal generator of a contraction semigroup of $C_{0}$-class in $X$, using the Theorem 1.4 we have the following theorem of local existence and uniqueness.

Theorem 4.1: Let $0 \leq \theta<\delta, 0 \leq \delta \leq 2,0 \leq \gamma \leq \frac{2+\delta}{2}, p>1$ integer and $0<n<8-2 \delta$. Then, for initial data $\left(u_{0}, u_{1}\right) \in H^{4-\delta}\left(\mathrm{R}^{\mathrm{n}}\right) \times H^{2}\left(\mathrm{R}^{\mathrm{n}}\right)$ there exist unique solution to the semilinear Cauchy Problem (19) defined in a maximal interval $\left[0, T_{\mathrm{m}}\right)$ in the class

$u \in C^{2}\left(\left[0, T_{m}\right), H^{\delta}\left(\mathbb{R}^{n}\right)\right) \cap C^{1}\left(\left[0, T_{m}\right), H^{2}\left(\mathbb{R}^{n}\right)\right) \cap C\left(\left[0, T_{m}\right), H^{4-\delta}\left(\mathbb{R}^{n}\right)\right)$

satisfying one and only one of the following conditions
(i) $T_{m}=\infty$
(ii) $T_{m}<\infty$ and $\lim _{t \rightarrow T_{m}}\|U\|_{X}+\left\|B_{1} U\right\|_{X}=\infty$.

4.1.2 Case $0 \leq \delta \leq \theta$ and $0 \leq \theta \leq \frac{2+\delta}{2}$

We write the system (19) in the standard matrix form as in previous section

$$
\left\{\begin{array}{l}
\frac{d U}{d t}=B_{2} U+F_{2}(U) \\
U(0)=U_{0}
\end{array}\right.
$$

where the operators $B_{2}: H^{A-\delta}\left(\mathrm{R}^{n}\right) \times H^{2}\left(\mathrm{R}^{n}\right) \rightarrow X$ and $F_{2}: D\left(B_{2}\right) \rightarrow$ $D\left(B_{2}\right)$ are given by

$$
B_{2}=\left(\begin{array}{cc}
0 & I \\
-A_{2} & -A_{\theta}
\end{array}\right) \quad \text { and } \quad F_{2}(U)=\left(\begin{array}{l}
0 \\
\left(I+(-\Delta)^{\delta}\right)^{-1}\left(u+v+\beta(-\Delta)^{\gamma} u^{p}\right)
\end{array}\right) .
$$

Similar to the previous section we prove, also in this case, that $F_{2}$ is well defined and is Lipschitz continuous on bounded sets of $D\left(B_{2}\right)$. In section 2 we proved that $B_{2}$ is infinitesimal generator ofa contraction semigroup of $C_{0}$-class in $X$. Then, using the Theorem 1.4 we obtain the local of existence and uniqueness as follows. 
Theorem 4.2: Let $0 \leq \delta \leq \theta, 0 \leq \theta \leq \frac{2+\delta}{2}, 0 \leq \gamma \leq \frac{2+\delta}{2}, p>1$ integer and $0<\mathrm{n}<8-2 \delta$. Then, for initial data $\left(u_{0}, u_{1}\right) \in H^{A-\delta}\left(\mathrm{R}^{\mathrm{n}}\right) \times H^{2}\left(\mathrm{R}^{\mathrm{n}}\right)$ there exist a unique solution to the semilinear Cauchy Problem (19) in a maximal interval $\left[0, T_{\mathrm{m}}\right)$ in the class

$$
u \in C^{2}\left(\left[0, T_{m}\right), H^{\delta}\left(\mathbb{R}^{n}\right)\right) \cap C^{1}\left(\left[0, T_{m}\right), H^{2}\left(\mathbb{R}^{n}\right)\right) \cap C\left(\left[0, T_{m}\right), H^{4-\delta}\left(\mathbb{R}^{n}\right)\right)
$$

such that one and only one of following conditions is true
i) $T_{m}=+\infty$
ii) $T_{m}<\infty$ and $\lim _{t \rightarrow T_{m}}\|U\|_{X}+\left\|B_{2} U\right\|_{X}=+\infty$.

\subsection{Global Existence}

In this section we show that the maximal interval of existence in the two previous cases is $[0, \infty)$. To do that, we assume $T_{m}<\infty$ and we claim that $\|U\|_{x}+\|B U\|_{x}<+\infty$. In such case we get $T_{m}=\infty$ and the global existence follows.

Taking the Fourier Transform in spatial variable $x$ on the Cauchy problem (19) we get the equivalent Cauchy problem in Fourier space

$$
\left\{\begin{array}{l}
\left(1+|\xi|^{2 \delta}\right) \hat{u}_{t t}+\left(\alpha|\xi|^{4}+|\xi|^{2}\right) \hat{u}+|\xi|^{2 \theta} \hat{u}_{t}=\beta|\xi|^{2 \gamma} \widehat{u^{p}} \\
\hat{u}(0, \xi)=\hat{u}_{0}(\xi) \\
\hat{u}_{t}(0, \xi)=\hat{u}_{1}(\xi) .
\end{array}\right.
$$

Using the Duhamel principle the solution of the Cauchy Problem 20 can be write as

$$
\hat{u}(t, \xi)=\hat{H}(t, \xi) \hat{u}_{0}+\hat{G}(t, \xi) \hat{u}_{1}+\beta \int_{0}^{t} \hat{G}(t-\tau, \xi) \frac{|\xi|^{2 \gamma}}{1+|\xi|^{2 \delta}} \widehat{u^{p}}(\tau, \xi) d \tau .
$$

Then, the derivative in time is given by

$$
\hat{u}_{t}(t, \xi)=\hat{H}_{t}(t, \xi) \hat{u}_{0}+\hat{G}_{t}(t, \xi) \hat{u}_{1}+\beta \int_{0}^{t} \hat{G}_{t}(t-\tau, \xi) \frac{|\xi|^{2 \gamma}}{1+|\xi|^{2 \delta}} \widehat{u^{p}}(\tau, \xi) d \tau,
$$

where the fundamental solutions to the linear problem are 


$$
\begin{gathered}
\hat{G}(t, \xi)=\frac{e^{\lambda_{+} t}-e^{\lambda_{-} t}}{\lambda_{+}-\lambda_{-}} \quad \text { and } \quad G(t, x)=\mathcal{F}^{-1}(\hat{G}(t, \cdot))(x), \\
\hat{H}(t, \xi)=\frac{\lambda_{+} e^{\lambda_{-} t}-\lambda_{-} e^{\lambda_{+} t}}{\lambda_{+}-\lambda_{-}} \quad \text { and } \quad H(t, x)=\mathcal{F}^{-1}(\hat{H}(t, \cdot))(x)
\end{gathered}
$$

and the associated characteristics roots are

$$
\lambda_{ \pm}=\frac{-|\xi|^{2 \theta} \pm \sqrt{|\xi|^{4 \theta}-4|\xi|^{2}\left(1+|\xi|^{2 \delta}\right)\left(1+\alpha|\xi|^{2}\right)}}{2\left(1+|\xi|^{2 \delta}\right)} .
$$

In the HORBACH, J. L., IKEHATA, R. e CHARÃO, R. C., 2016 is calculated in Lemma 3.6 the following estimate to solutions of the linear problem.

$$
\begin{aligned}
& \left(1+|\xi|^{2 \delta}\right)\left|\hat{u}_{t}\right|^{2}+|\xi|^{2}\left(1+\alpha|\xi|^{2}\right)|\hat{u}|^{2} \leq 5 e^{-\frac{1}{5} \rho_{\theta}(\xi) t}\left(\left(1+|\xi|^{2 \delta}\right)\left|\hat{u}_{1}\right|^{2}+|\xi|^{2}\left(1+\alpha|\xi|^{2}\right)\left|\hat{u}_{0}\right|^{2}\right) \\
& \text { where } \rho_{\theta}(\xi)=\left\{\begin{array}{cllc}
\varepsilon|\xi|^{2-2 \theta}\left(1+\alpha|\xi|^{2}\right), & |\xi| \leq 1 & \text { e } & 0 \leq \theta \leq \frac{1}{2} \\
\varepsilon \frac{|\xi|^{2 \theta}}{1+|\xi|^{2 \delta}}, & |\xi| \leq 1 & \text { e } & \frac{1}{2}<\theta \leq \frac{2+\delta}{2} \\
\varepsilon \frac{|\xi|^{2 \theta}}{1+|\xi|^{2 \delta}}, & |\xi| \geq 1 & \text { e } & 0 \leq \theta \leq \frac{2+\delta}{2}
\end{array}\right.
\end{aligned}
$$

We use the estimate (23) to prove the next lemma.

Lemma 4.4: Let $\hat{G}(t, \xi)$ and $\hat{H}(t, \xi)$ fundamental solutions of linear problem associated to Problem (20). Then we have the following estimates:
i) $\left|\hat{G}_{t}\right|^{2} \leq 5 e^{-\frac{1}{5} \rho_{\theta}(\xi) t}$
iii) $\left|\hat{H}_{t}\right|^{2} \leq 5 e^{-\frac{1}{5} \rho_{\theta}(\xi) t} \frac{|\xi|^{2}\left(1+\alpha|\xi|^{2}\right)}{\left(1+|\xi|^{2 \delta}\right)} ;$
ii) $|\hat{G}|^{2} \leq 5 e^{-\frac{1}{5} \rho_{\theta}(\xi) t} \frac{\left(1+|\xi|^{2 \delta}\right)}{|\xi|^{2}\left(1+\alpha|\xi|^{2}\right)} ;$
iv) $|\hat{H}|^{2} \leq 5 e^{-\frac{1}{5} \rho_{\theta}(\xi) t}$.

Proof: To show items (i) and (ii) we consider the solution of the homogeneous problem (20) with $\hat{u}_{0}=0$. Then, we have $\hat{u}(t, \xi)=\hat{G}(t$,

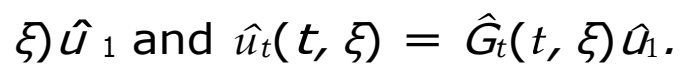

Substituting these expressions on the left hand side of the estimate (23) the result for (i) and (ii) follows. The proof of item (iii) and (iv) is similar. 
Now, to prove the claim that $\|U\|_{x}+\|B U\|_{x}<+\infty$

we need consider again the two cases on $\delta$ and $\theta$.

\subsubsection{Case $0 \leq \theta<\delta$ and $0 \leq \delta \leq 2$}

We need in this case to show that the norm $\|U(t)\|_{x}+\| B_{1} U(t)$ $\|_{X}$ is bounded for all $t \in\left[0, T_{m}\right)$ by assuming that $T_{m}<+\infty$.

By definition of $B\left(u, u_{t}\right)=\left(u_{t},-A_{2} u\right)$, where $A_{2}=\left(I+(-\Delta)^{\text {o }}\right)$ ${ }^{-1}\left(a \Delta^{2}-\Delta+I\right)$, we have

$$
\begin{aligned}
& \|U\|_{X}^{2}+\left\|B_{1} U\right\|_{X}^{2}=\|u\|_{H^{2}}^{2}+\left\|u_{t}\right\|_{H^{\delta}}^{2}+\left\|u_{t}\right\|_{H^{2}}^{2}+\left\|A_{2} u\right\|_{H^{\delta}}^{2} \\
& =\int_{\mathbb{R}^{n}}\left(1+|\xi|^{2}+\alpha|\xi|^{4}\right)|\hat{u}|^{2} d \xi+\int_{\mathbb{R}^{n}}\left(1+|\xi|^{2 \delta}\right)\left|\widehat{A_{2} u}\right|^{2} d \xi \\
& +\int_{\mathbb{R}^{n}}\left(1+|\xi|^{2}+\alpha|\xi|^{4}\right)\left|\hat{u}_{t}\right|^{2} d \xi+\int_{\mathbb{R}^{n}}\left(1+|\xi|^{2 \delta}\right)\left|\hat{u}_{t}\right|^{2} d \xi \\
& =\int_{\mathbb{R}^{n}} \frac{\left(1+|\xi|^{2}+\alpha|\xi|^{4}\right)}{\left(1+|\xi|^{2 \delta}\right)}\left(2+|\xi|^{2 \delta}+|\xi|^{2}+\alpha|\xi|^{4}\right)|\hat{u}|^{2} d \xi+\int_{\mathbb{R}^{n}}\left(2+|\xi|^{2 \delta}+|\xi|^{2}+\alpha|\xi|^{4}\right)\left|\hat{u}_{t}\right|^{2} d \xi .
\end{aligned}
$$

We note that

$$
\frac{\left(1+|\xi|^{2}+\alpha|\xi|^{4}\right)}{\left(1+|\xi|^{2 \delta}\right)}\left(2+|\xi|^{2 \delta}+|\xi|^{2}+\alpha|\xi|^{4}\right) \leq C\left(1+|\xi|^{2(4-\delta)}\right)
$$

and

$\left(2+|\xi|^{2 \delta}+|\xi|^{2}+a|\xi|^{4}\right) \leq C 1+|\xi|^{4}$

for all $\xi \in \mathrm{Rn}$, Then, we obtain

$$
\|U\|_{X}^{2}+\left\|B_{1} U\right\|_{X}^{2} \leq C \int_{\mathbb{R}^{n}}\left(1+|\xi|^{2(4-\delta)}\right)|\hat{u}|^{2} d \xi+C \int_{\mathbb{R}^{n}}\left(1+|\xi|^{4}\right)\left|\hat{u}_{t}\right|^{2} d \xi .
$$

Substituting $\hat{u}$ and $a_{t}$, given by (21) and (22) respectively, in the above inequality we have

$$
\begin{aligned}
& \|U\|_{X}^{2}+\left\|B_{1} U\right\|_{X}^{2} \leq C \int_{\mathbb{R}^{n}}\left(\left(1+|\xi|^{2(4-\delta)}\right)|\hat{H}|^{2}+\left(1+|\xi|^{4}\right)\left|\hat{H}_{t}\right|^{2}\right)\left|\hat{u}_{0}\right|^{2} d \xi \\
& +C \int_{\mathbb{R}^{n}}\left(\left(1+|\xi|^{2(4-\delta)}\right)|\hat{G}|^{2}+\left(1+|\xi|^{4}\right)\left|\hat{G}_{t}\right|^{2}\right)\left|\hat{u}_{1}\right|^{2} d \xi \\
& +C \int_{0}^{t} \int_{\mathbb{R}^{n}}\left(1+|\xi|^{2(4-3 \delta)}\right)|\xi|^{4 \gamma}|\hat{G}|^{2}\left|\widehat{u^{p}}\right|^{2} d \xi d \tau+C \int_{0}^{t} \int_{\mathbb{R}^{n}}\left(1+|\xi|^{2(2-2 \delta)}\right)|\xi|^{4 \gamma}\left|\hat{G}_{t}\right|^{2}\left|\widehat{u^{p}}\right|^{2} d \xi d \tau .
\end{aligned}
$$


Now, using the estimates in Lemma 4.4 and the fact that $e^{-\frac{1}{5} \rho_{\theta}(\xi) t}$ $\leq 1$ we obtain

$$
\begin{aligned}
& \|U\|_{X}^{2}+\left\|B_{1} U\right\|_{X}^{2} \leq C \int_{\mathbb{R}^{n}}\left(\left(1+|\xi|^{2(4-\delta)}\right)+\left(1+|\xi|^{4}\right) \frac{|\xi|^{2}\left(1+\alpha|\xi|^{2}\right)}{\left(1+|\xi|^{2 \delta}\right)}\right)\left|\hat{u}_{0}\right|^{2} d \xi \\
& +C \int_{\mathbb{R}^{n}}\left(\left(1+|\xi|^{2(4-\delta)}\right) \frac{\left(1+|\xi|^{2 \delta}\right)}{|\xi|^{2}\left(1+\alpha|\xi|^{2}\right)}+\left(1+|\xi|^{4}\right)\right)\left|\hat{u}_{1}\right|^{2} d \xi \\
& +C \int_{0}^{t} \int_{\mathbb{R}^{n}}\left(\left(1+|\xi|^{2(4-3 \delta)}\right)|\xi|^{4 \gamma} \frac{\left(1+|\xi|^{2 \delta}\right)}{|\xi|^{2}\left(1+\alpha|\xi|^{2}\right)}\left|\widehat{u^{p}}\right|^{2}+\left(1+|\xi|^{2(2-2 \delta)}\right)|\xi|^{4 \gamma}\left|\widehat{u^{p}}\right|^{2}\right) d \xi d \tau .
\end{aligned}
$$

We observe that in the second integral at the right hand side of (24) $\hat{u}_{1}$ appears a singularity given by $|\xi|^{-2}$. So, this term is a little delicate to deal with in the zone of low frequency, that is, $\xi$ near zero. To do that we assume additional regularity on the initial data $u_{1}$. In the third integral we have the same singularity but it is controlled by the term $\mid \xi^{4 y}$ from the nonlinearity because $r \geq 1 / 2$.

Note that, for $|\xi| \geq 1$ we have

$$
\left(1+|\xi|^{2(4-\delta)}\right) \frac{\left(1+|\xi|^{2 \delta}\right)}{|\xi|^{2}\left(1+\alpha|\xi|^{2}\right)} \leq C\left(1+|\xi|^{4}\right)
$$

and for $0<|\xi| \leq 1$ we have

$$
\left(1+|\xi|^{2(4-\delta)}\right) \frac{\left(1+|\xi|^{2 \delta}\right)}{|\xi|^{2}\left(1+\alpha|\xi|^{2}\right)} \leq 4|\xi|^{-2}
$$

Using these estimates, we estimate the integral where appears the initial data $\hat{u}_{1}$ em (24) working at the zones of low and high frequency. The integral on high frequency is estimate by $\left\|u_{1}\right\|_{H^{2}}^{2}$ and the integral on the low frequency is estimate by the norm of $u_{1}$ in $\dot{W}^{-1,1}\left(R^{n}\right)$. The other integrals at the right hand side of (24) can be estimate in standard way. Therefore, we conclude that 


$$
\begin{aligned}
\|U\|_{X}^{2}+\left\|B_{1} U\right\|_{X}^{2} & \leq C\left\|u_{0}\right\|_{H^{4-\delta}}^{2}+C\left\|u_{1}\right\|_{H^{2}}^{2}+C \int_{|\xi| \leq 1}|\xi|^{-2}\left|\hat{u}_{1}\right|^{2} d \xi \\
& +C \int_{0}^{t} \int_{\mathbb{R}^{n}}\left(1+|\xi|^{2(2-2 \delta+2 \gamma)}\right)\left|\widehat{u^{p}}\right|^{2} d \xi d \tau \\
& \leq C\left\|u_{0}\right\|_{H^{4-\delta}}^{2}+C\left\|u_{1}\right\|_{H^{2}}^{2}+C\left\|u_{1}\right\|_{\dot{W}^{-1,1}}^{2}+C \int_{0}^{t}\left\|u^{p}\right\|_{H^{2-2 \delta+2 \gamma}}^{2} d \tau \\
& \leq C\left\|u_{0}\right\|_{H^{4-\delta}}^{2}+C\left\|u_{1}\right\|_{H^{2}}^{2}+C\left\|u_{1}\right\|_{\dot{W}^{-1,1}}^{2}+C \int_{0}^{t}\left\|u^{p}\right\|_{H^{4-\delta}}^{2} d \tau .
\end{aligned}
$$

Using the Lemma 1.3 with $0<n<8-2 \delta$ we have for $p>1$ integer

$$
\begin{aligned}
& \|U(t)\|_{X}^{2}+\left\|B_{1} U(t)\right\|_{X}^{2} \leq C\left\|u_{0}\right\|_{H^{4-\delta}}^{2}+C\left\|u_{1}\right\|_{H^{2}}^{2}+C\left\|u_{1}\right\|_{W^{-1,1}}^{2}+C \int_{0}^{t}\|u\|_{H^{4-\delta}}^{2 p} d \tau \\
& \leq C\left\|u_{0}\right\|_{H^{4-\delta}}^{2}+C\left\|u_{1}\right\|_{H^{2}}^{2}+\left\|u_{1}\right\|_{\dot{W}^{-1,1}}^{2}+T_{m} \sup _{0 \leq \tau \leq t}\|u(\tau)\|_{H^{4-\delta}}^{2 p} \\
& \leq C\left\|B_{1} U_{0}\right\|_{X}^{2}+C\left\|u_{1}\right\|_{\dot{W}^{-1,1}}^{2}+C T_{m} \sup _{0 \leq \tau \leq t}\left\|B_{1} U(\tau)\right\|_{X}^{2 p}
\end{aligned}
$$

for all $t \in\left[0, T_{m}\right)$ with the maximum time of existence $T_{m}$ is assumed to be finite.

Now, we define the function

$$
M_{1}(t)=\sup _{0 \leq \tau \leq t}\left(\|U(\tau)\|_{X}^{2}+\left\|B_{1} U(\tau)\right\|_{X}^{2}\right) \text { for } 0 \leq t \leq T_{m}
$$

From the previous inequality we get that $M_{1}(t)$ satisfies

$$
M_{1}(t) \leq C\left(\left\|B_{1} U_{0}\right\|_{X}^{2}+\left\|u_{1}\right\|_{\dot{W}^{-1,1}}^{2}\right)+C T_{m} M_{1}(t)^{p}, \quad \forall t \in\left[0, T_{m}\right) \text { with } T_{m}<+\infty .
$$

In order to show that the solution obtained to the Cauchy Problem (19) is global, that is $T_{m}=+\infty$, we need the next elementary lemma of calculus.

Lemma 4.5: Let $p>1$ and $F(M)=a I_{0}+b T M^{p}-M$ a continuous positive function on $M \geq 0$, with $a, b, I_{0}, T$ positive constants. Then, there exist a unique $M_{0}>0$ absolute minimum point of $F(M)$ in $[0, \infty)$. In addition, there exist $\varepsilon>0$ such that $F\left(M_{0}\right)<0$ if $0<I_{0} \leq \varepsilon$. 
We note that the function $M_{1}(t)$ is not negative and satisfy $F$ $\left(M_{1}(t)\right) \geq 0$ for all $t \in\left[0, T_{m}\right)$ due to inequality (26) with $F(M)$ the function given in Lemma 4.5 with

$$
a=b=C, T=T_{m} \text { and } I_{0}=\left\|B_{1} U_{0}\right\|_{X}^{2}+\left\|u_{1}\right\|_{\dot{W}^{-1,1}}^{2} .
$$

Therefore, if $0<I_{0} \leq \varepsilon, \varepsilon>0$ given by Lemma 4.5, due to the continuity of the function $M_{1}(t)$, there are only two possibilities:
(i) $M_{1}(t)<M_{0}$, for all $t \in\left[0, T_{m}\right)$
or
(ii) $M_{1}(t)>M_{0}$, for all $t \in\left[0, T_{m}\right)$.

However, we note that

$$
M_{1}(0)=\left\|U_{0}\right\|_{X}^{2}+\left\|B_{1} U_{0}\right\|_{X}^{2}
$$

Then, assuming another condition on the initial data that $M_{1}(0)<$ $M_{0}$ ( $M_{0}$ the global minimum point in Lemma 4.5) it follows that $M_{1}(t) \leq$ $M_{0}$ for all $t \in\left[0, T_{m}\right)$. Them the condition that holds is (i). Therefore, if $T_{m}$ is finite, we have proved that

$$
\|U\|_{X}^{2}+\left\|B_{1} U\right\|_{X}^{2}
$$

is bounded for all $t \in\left[0, T_{m}\right)$. This contradicts the condition of Theorem 4.1. Then, we must have $T_{m}=\infty$ and the solution is global for the case in consideration. The result is

Theorem 4.3: Let $0 \leq \theta<\delta, 0 \leq \delta \leq 2,1 \leq \gamma \leq \frac{2+\delta}{2}, p>1$ integer and 1 $\leq n<8-2 \delta$. Consider the initial data $u_{0} \in H^{A-\delta}\left(\mathrm{R}^{\mathrm{n}}\right)$ and $u_{1} \in H^{2}\left(\mathrm{R}^{\mathrm{n}}\right) \cap \dot{W}$ ${ }^{-1,1}\left(\mathrm{R}^{n}\right)$ satisfying $0<I_{0} \leq \varepsilon$ and $M_{1}(0)<M_{0}$ with $\varepsilon, I_{0}, M_{0}, M_{1}(0)$ given above and in Lemma 4.5.

Then, there exist a unique global solution $u=u(t, x)$ to the Cauchy Problem (19) such that

$$
u \in C^{2}\left([0, \infty), H^{\delta}\left(\mathbb{R}^{n}\right)\right) \cap C^{1}\left([0, \infty), H^{2}\left(\mathbb{R}^{n}\right)\right) \cap C\left([0, \infty), H^{4-\delta}\left(\mathbb{R}^{n}\right)\right) .
$$


4.2.2 Case $0 \leq \delta \leq \theta$ and $0 \leq \theta \leq \frac{2+\delta}{2}$

To this case, we need to find a upper bound for the norm $\|U\|_{X}+\| B_{2} U$ II $x$ for all $t \in\left[0, T_{m}\right)$, with $U=\left(u, u_{t}\right)$ where $u$ is the solution of (19) given by Theorem 4.2. Analogously to the previous section we may obtain such estimate. This fact proves that the solution is global and the following result holds.

Theorem 4.4: Let $0 \leq \delta \leq \theta, 0 \leq \theta \leq \frac{2+\delta}{2}, \frac{1}{2} \leq \gamma \leq \frac{2+\delta}{2}, p>1$ integer and $1 \leq n<8-2 \delta$. Consider the initial data $u_{0} \in H^{4-\delta}\left(\mathrm{R}^{n}\right)$ and $u_{1} \in H^{2}\left(\mathrm{R}^{n}\right) \cap$ $\dot{W}^{-1,1}\left(\mathrm{R}^{n}\right)$ satisfying $0<I_{0} \leq \varepsilon$ and $M_{2}(0)<M_{0}$ with $\varepsilon_{,} I_{0}, M_{0}, M_{2}(0)$ given in a similar way as in previous case. Then, for this case on $\delta$ and $\theta$, there exist unique global solution $u=u(t, x)$ to the Cauchy Problem (19) such that

$$
u \in C^{2}\left([0, \infty), H^{\delta}\left(\mathbb{R}^{n}\right)\right) \cap C^{1}\left([0, \infty), H^{2}\left(\mathbb{R}^{n}\right)\right) \cap C\left([0, \infty), H^{4-\delta}\left(\mathbb{R}^{n}\right)\right) .
$$

\section{DECAY RATES: SEMILINEAR PROBLEMA}

From Theorems 4.3 and 4.4 the semilinear Problem (19) has a unique global solution in the class

$$
u \in C^{2}\left([0, \infty), H^{\delta}\left(\mathbb{R}^{n}\right)\right) \cap C^{1}\left([0, \infty), H^{2}\left(\mathbb{R}^{n}\right)\right) \cap C\left([0, \infty), H^{4-\delta}\left(\mathbb{R}^{n}\right)\right)
$$

for all $0 \leq \delta \leq 2,0 \leq \theta \leq \frac{2+\delta}{2}, \frac{1}{2} \leq \gamma \leq \frac{2+\delta}{2}, p>1$ integer and $1 \leq n<$ $8-2 \delta$. Consider the initial data $u_{0} \in H^{4-\delta}\left(\mathrm{R}^{n}\right)$ and $u_{1} \in H^{2}\left(\mathrm{R}^{n}\right) \cap \dot{W}^{-1,1}\left(\mathrm{R}^{n}\right)$ small enough.

In this section we show decay rates to the energy and $L^{2}\left(R^{n}\right)$ norm of the solution to the semilinear problem (19) by using estimates similar to the estimates in previous sections. 
We note that is sufficient to get estimates for the norm \| $\left(u, u_{t}\right) \|$ $H^{4-\delta} \times H^{2}$ to obtain decay rates to the energy norm and $L^{2}\left(R^{n}\right)$-norm. In fact, it holds that

$$
\begin{aligned}
& \int_{\mathbb{R}^{n}}\left[\left(1+|\xi|^{2 \delta}\right)\left|\hat{u}_{t}\right|^{2}+|\xi|^{2}\left(1+\alpha|\xi|^{2}\right)|\hat{u}|^{2}+|\hat{u}|^{2}\right] d \xi \\
& \leq \int_{|\xi| \leq 1}\left[\left(2+|\xi|^{2}+|\xi|^{4}\right)\left|\hat{u}_{t}\right|^{2}+(1+\alpha)\left(1+|\xi|^{2(4-\delta)}\right)|\hat{u}|^{2}\right] d \xi \leq C\left\|\left(u, u_{t}\right)\right\|_{H^{4-\delta} \times H^{2}}^{2} .
\end{aligned}
$$

Let us now find an estimate for $\left\|\left(u, u_{t}\right)\right\|_{H^{4-\delta} \times H^{2}}$. In section 4.2 we have expressions for the solution $\hat{u}$ and its derivative $\hat{u}_{t}$ (see (21) and (22)). Then, using such expressions and the definition of norm $H^{4-\delta} \times H^{2}$ we obtain

$$
\begin{aligned}
& \left\|\left(u, u_{t}\right)\right\|_{H^{4-\delta} \times H^{2}}^{2} \leq C \int_{\mathbb{R}^{n}}\left(\left(1+|\xi|^{2(4-\delta)}\right)|\hat{H}|^{2}+\left(1+|\xi|^{2}+\alpha|\xi|^{4}\right)\left|\hat{H}_{t}\right|^{2}\right)\left|\hat{u}_{0}\right|^{2} d \xi \\
& +C \int_{\mathbb{R}^{n}}\left(\left(1+|\xi|^{2(4-\delta)}\right)|\hat{G}|^{2}+\left(1+|\xi|^{2}+\alpha|\xi|^{4}\right)\left|\hat{G}_{t}\right|^{2}\right)\left|\hat{u}_{1}\right|^{2} d \xi \\
& +C \int_{0}^{t} \int_{\mathbb{R}^{n}}\left(1+|\xi|^{2(4-\delta)}\right) \frac{|\xi|^{4 \gamma}}{\left(1+|\xi|^{2 \delta}\right)^{2}}|\hat{G}(t-\tau)|^{2}\left|\widehat{u^{p}}\right|^{2} d \xi d \tau \\
& +C \int_{0}^{t} \int_{\mathbb{R}^{n}}\left(1+|\xi|^{2}+\alpha|\xi|^{4}\right) \frac{|\xi|^{4 \gamma}}{\left(1+|\xi|^{2 \delta}\right)^{2}}\left|\hat{G}_{t}(t-\tau)\right|^{2}\left|\widehat{u^{p}}\right|^{2} d \xi d \tau .
\end{aligned}
$$

Now, by considering the estimates in Lemma 4.4 we arrive at the estimate

$$
\begin{aligned}
& \left\|\left(u, u_{t}\right)\right\|_{H^{4-\delta} \times H^{2}}^{2} \leq C \int_{\mathbb{R}^{n}} e^{-\frac{1}{5} \rho_{\theta}(\xi) t}\left(\left(1+|\xi|^{2(4-\delta)}\right)+\left(1+|\xi|^{2}+\alpha|\xi|^{4}\right) \frac{\left.\xi\right|^{2}\left(1+\alpha|\xi|^{2}\right)}{\left(1+|\xi|^{2 \delta}\right)}\right)\left|\hat{u}_{0}\right|^{2} d \xi \\
& +C \int_{\mathbb{R}^{n}} e^{-\frac{1}{5} \rho_{\theta}(\xi) t}\left(\left(1+|\xi|^{2(4-\delta)}\right) \frac{\left(1+|\xi|^{2 \delta}\right)}{|\xi|^{2}\left(1+\alpha|\xi|^{2}\right)}+\left(1+|\xi|^{2}+\alpha|\xi|^{4}\right)\right)\left|\hat{u}_{1}\right|^{2} d \xi \\
& +C \int_{0}^{t} \int_{\mathbb{R}^{n}} e^{-\frac{t}{\rho} \rho_{0}(\xi)(t-\tau)}\left(1+|\xi|^{2(4-\delta)}\right) \frac{|\xi|^{4 \gamma}}{|\xi|^{2}\left(1+\alpha|\xi|^{2}\right)\left(1+|\xi|^{2 \delta}\right)}\left|\hat{u}^{p}\right|^{2} d \xi d \tau \\
& +C \int_{0}^{t} \int_{\mathbb{R}^{n}} e^{-\frac{1}{5} \rho_{0}(\xi)(t-\tau)}\left(1+|\xi|^{2}+\alpha|\xi|^{4}\right) \frac{|\xi|^{4 \gamma}}{\left(1+|\xi|^{2 \delta}\right)^{2}}\left|\hat{p}^{p}\right|^{2} d \xi d \tau .
\end{aligned}
$$

We note here that the terms that appear in the above inequality can be estimated for, $0 \leq \delta \leq 2,0 \leq \theta \leq \frac{2+\delta}{2}$ and $\frac{1}{2} \leq \gamma \leq \frac{2+\delta}{2}$, for all $\varepsilon \in \mathrm{R}^{n}$, as follows 

i) $\left(1+|\xi|^{2(4-\delta)}\right)+\left(1+|\xi|^{2}+\alpha|\xi|^{4}\right) \frac{|\xi|^{2}\left(1+\alpha|\xi|^{2}\right)}{\left(1+|\xi|^{2 \delta}\right)} \leq C\left(1+|\xi|^{2(4-\delta)}\right)$;
ii) $\left(1+|\xi|^{2(4-\delta)}\right) \frac{|\xi|^{4 \gamma}}{|\xi|^{2}\left(1+\alpha|\xi|^{2}\right)\left(1+|\xi|^{2 \delta}\right)} \leq C\left(1+|\xi|^{2(2+2 \gamma-2 \delta)}\right), \quad\left(\gamma \geq \frac{1}{2}\right)$;
iii) $\left(1+|\xi|^{2}+\alpha|\xi|^{4}\right) \frac{|\xi|^{4 \gamma}}{\left(1+|\xi|^{2 \delta}\right)^{2}} \leq C\left(1+|\xi|^{2(2+2 \gamma-2 \delta)}\right)$.

As in (24) we can observe that in the estimate (ii) above appears the singularity $|\xi|^{-2}$ for $\xi$ near zero which is controlled by the term $|\xi|^{4} r$ since we have assumed $\gamma \geq 1 / 2$ On the second integral at the right hand side of the last estimate, as in (24), we do not have the term $\mid \xi^{4 r}$ because that we assume the additional hypotheses $u_{1} \in \dot{W}^{-1,1}\left(R^{n}\right)$. The problem with such singularity is at the zones of low frequency. The integral on high frequency can be estimate in standard way.

In fact, to estimate the coefficient of $\left|\hat{u}_{1}\right|^{2}$, we see that for $|\xi| \geq 1$

$$
\left(1+|\xi|^{2(4-\delta)}\right) \frac{\left(1+|\xi|^{2 \delta}\right)}{|\xi|^{2}\left(1+\alpha|\xi|^{2}\right)} \leq C\left(1+|\xi|^{2}+\alpha|\xi|^{4}\right)
$$

because $|\xi|^{2}\left(1+a|\xi|^{2}\right) \geq\left(1+a|\xi|^{4}\right)$.

For $0<|\xi| \leq 1$ we have $\left(1+|\xi|^{2(4-\delta)}\right) \frac{\left(1+|\xi|^{2 \delta}\right)}{|\xi|^{2}\left(1+\alpha|\xi|^{2}\right)} \leq 4|\xi|^{-2}$

tghuis, using the above estimates, we may conclude that $\|\left(u, u_{t}\right)$ II ${ }_{H^{4}-\delta \times H^{2}}$ is bounded by the four integrals as shown below

$$
\begin{aligned}
& \left\|\left(u, u_{t}\right)\right\|_{H^{4-\delta} \times H^{2}}^{2} \leq C \int_{\mathbb{R}^{n}} e^{-\frac{1}{5} \rho_{\theta}(\xi) t}\left(1+|\xi|^{2(4-\delta)}\right)\left|\hat{u}_{0}\right|^{2} d \xi+C \int_{\mathbb{R}^{n}} e^{-\frac{1}{5} \rho_{\theta}(\xi) t}\left(1+|\xi|^{2}+\alpha|\xi|^{4}\right)\left|\hat{u}_{1}\right|^{2} d \xi \\
& +C \int_{|\xi| \leq 1} e^{-\frac{1}{5} \rho_{\theta}(\xi) t}|\xi|^{-2}\left|\hat{u}_{1}\right|^{2} d \xi+C \int_{0}^{t} \int_{\mathbb{R}^{n}} e^{-\frac{1}{5} \rho_{\theta}(\xi)(t-\tau)}\left(1+|\xi|^{2+2 \gamma-2 \delta}\right)\left|\widehat{u}^{p}\right|^{2} d \xi d \tau .
\end{aligned}
$$

At this point, we define the following integrals, dependent on $t$, which appear in the above estimate 
- $L_{1}(t)=C \int_{\mathbb{R}^{n}} e^{-\frac{1}{5} \rho_{\theta}(\xi) t}\left(1+|\xi|^{2(4-\delta)}\right)\left|\hat{u}_{0}\right|^{2} d \xi$

- $L_{2}(t)=C \int_{\mathbb{R}^{n}} e^{-\frac{1}{5} \rho_{\theta}(\xi) t}\left(1+|\xi|^{2}+\alpha|\xi|^{4}\right)\left|\hat{u}_{1}\right|^{2} d \xi$

- $L_{3}(t)=C \int_{|\xi| \leq 1} e^{-\frac{1}{5} \rho_{\theta}(\xi) t}|\xi|^{-2}\left|\hat{u}_{1}\right|^{2} d \xi$

- $N_{1}(t)=C \int_{0}^{t} \int_{\mathbb{R}^{n}} e^{-\frac{1}{5} \rho_{\theta}(\xi)(t-\tau)}\left(1+|\xi|^{2(2+2 \gamma-2 \delta)}\right)\left|\widehat{u}^{p}\right|^{2} d \xi d \tau$.

The function $\rho_{\theta}$ define in section 3 depends on $\theta$, then we separated the problem into four cases:

i) Case $0 \leq \delta \leq \theta$ and $0 \leq \theta \leq \frac{1}{2}$;

ii) Case $0 \leq \delta \leq \theta$ and $\frac{1}{2}<\theta \leq \frac{2+\delta}{2}$;

iii) Case $0 \leq \theta<\delta \leq 2$ and $0 \leq \theta \leq \frac{1}{2}$ iv) Case $0 \leq \theta<\delta \leq 2$ and $\frac{1}{2}<\theta \leq \frac{2+\delta}{2}$.

In the next subsections we show decay estimates to the energy and for the $L^{2}$-norm of the solution in cases (i) and (ii). These estimates refer to the case $0 \leq \delta \leq \theta$ where we do not need to impose more regularity on the initial data compared to the decay rates was already obtained to the liner problem. The cases (iii) and (iv) can be estimated in the same way but assuming regularity on the initial data.

\subsection{Case $0 \leq \delta \leq \theta$ and $0 \leq \theta \leq \frac{1}{2}$}

In this subsection we find decay rates to the $L^{2}$-norm and to the energy for the semilinear problem.

For the case $0 \leq \theta \leq \frac{1}{2}$ we have $\rho_{\theta}(\xi)=\left\{\begin{array}{clll}\varepsilon|\xi|^{2-2 \theta}\left(1+\alpha|\xi|^{2}\right), & |\xi| \leq 1 & \text { e } & 0 \leq \theta \leq \frac{1}{2} \\ \varepsilon \frac{|\xi|^{2 \theta}}{1+|\xi|^{2 \delta}}, & |\xi| \geq 1 & \text { e } & 0 \leq \theta \leq \frac{1}{2}\end{array}\right.$

Since $\rho_{\theta}=\rho_{\theta}(\xi)$ also depends on $\xi$, we estimate $e^{-\frac{1}{5} \rho_{\theta} t}$ in the low and high frequency in the following way 


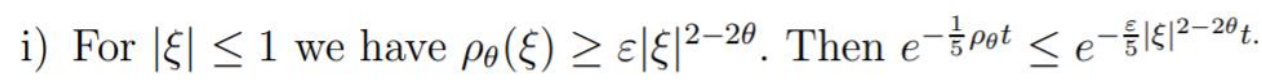

ii) For $|\xi| \geq 1$ we have $\rho_{\theta}(\xi) \geq \frac{\varepsilon}{2}$ because we are considering $\theta \geq \delta$. Thus, we also have $e^{-\frac{1}{5} \rho_{\theta} t} \leq e^{-\frac{\varepsilon}{10} t}$ in this case.

Lemma 5.1: Letp $>1$ integer and $1 \leq n<8-2 \delta$. Let $\theta, \delta$ and $y$ such that $0 \leq \delta \leq \theta, 0 \leq \theta \leq \frac{1}{2}, \frac{1}{2} \leq \gamma \leq \frac{2+\delta}{2}$. Then, for all initial data $u_{0} \in$ $H^{A-\delta}\left(\mathrm{R}^{n}\right) \cap L^{1}\left(\mathrm{R}^{n}\right)$ and $u_{1} \in H^{2}\left(\mathrm{R}^{n}\right) \cap L^{1}\left(\mathrm{R}^{n}\right) \cap \dot{W}^{-1,1}\left(\mathrm{R}^{n}\right)$ we have

$$
\begin{aligned}
\left\|\left(u, u_{t}\right)\right\|_{H^{4-\delta} \times H^{2}}^{2} & \leq C\left(\left\|\left(u_{0}, u_{1}\right)\right\|_{L^{1} \times L^{1}}^{2}+\left\|u_{1}\right\|_{\dot{W}^{-1,1}}^{2}+\left\|\left(u_{0}, u_{1}\right)\right\|_{H^{4-\delta} \times H^{2}}^{2}\right)(1+t)^{-\frac{n}{2-2 \theta}} \\
& +C \int_{0}^{t}\left\|\left(u, u_{t}\right)\right\|_{H^{4-\delta} \times H^{2}}^{2 p}(1+t-\tau)^{-\frac{n}{2-2 \theta}} d \tau, \quad \forall t>0 .
\end{aligned}
$$

Proof: We start estimating $L_{1}$ defined above. First we separated the integral into two integrals, at the low frequency $(|\xi| \leq 1)$ and another at the high frequency $(|\xi| \geq 1)$. Then we get

$$
\begin{aligned}
& L_{1}=C \int_{\mathbb{R}^{n}} e^{-\frac{1}{5} \rho_{\theta}(\xi) t}\left(1+|\xi|^{2(4-\delta)}\right)\left|\hat{u}_{0}\right|^{2} d \xi \\
& \leq C \int_{|\xi| \leq 1} e^{-\frac{\varepsilon}{5}|\xi|^{2-2 \theta} t}\left(1+|\xi|^{2(4-\delta)}\right)\left|\hat{u}_{0}\right|^{2} d \xi+C \int_{|\xi| \geq 1} e^{-\frac{\varepsilon}{10} t}\left(1+|\xi|^{2(4-\delta)}\right)\left|\hat{u}_{0}\right|^{2} d \xi .
\end{aligned}
$$

We use the Lemma 1.8 to estimate the integral at the low frequency and the definition of norm in $H^{s}\left(\mathrm{R}^{n}\right)$ to estimate the integral at the high frequency. Thus we obtain

$$
\begin{aligned}
& L_{1} \leq C\left\|u_{0}\right\|_{L^{1}}^{2} \int_{|\xi| \leq 1} e^{-\frac{\varepsilon}{5}|\xi|^{2-2 \theta}} d \xi+C e^{-\frac{\varepsilon}{10} t} \int_{|\xi| \geq 1}\left(1+|\xi|^{2(4-\delta)}\right)\left|\hat{u}_{0}\right|^{2} d \xi \\
& \leq C\left\|u_{0}\right\|_{L^{1}}^{2}(1+t)^{-\frac{n}{2-2 \theta}}+C e^{-\frac{\varepsilon}{10} t}\left\|u_{0}\right\|_{H^{4-\delta}}^{2} \leq C\left(\left\|u_{0}\right\|_{L^{1}}^{2}+\left\|u_{0}\right\|_{H^{4-\delta}}^{2}\right)(1+t)^{-\frac{n}{2-2 \theta}}, \quad \forall t>0 .
\end{aligned}
$$

In the same way we can easily estimate $L_{2}$ to get

$$
L_{2} \leq C\left(\left\|u_{1}\right\|_{L^{1}}^{2}+\left\|u_{1}\right\|_{H^{2}}^{2}\right)(1+t)^{-\frac{n}{2-2 \theta}}, \quad \forall t>0
$$


The estimate for $\mathrm{L} 3$ follows from the definition of the Sobolev space $\dot{W}$ ${ }^{-1,1}\left(R^{n}\right)$ and from Lemma 1.8. Then we have for $t>0$

To get an estimate to $N_{1}$ we again estimate the associated integral into low frequency and high frequency as follows

$$
\begin{aligned}
N_{1} & =C \int_{0}^{t} \int_{\mathbb{R}^{n}} e^{-\frac{1}{5} \rho_{\theta}(t-\tau)}\left(1+|\xi|^{2(2+2 \gamma-2 \delta)}\right)\left|\widehat{u^{p}}\right|^{2} d \xi d \tau \\
& \leq C \int_{0}^{t} \int_{|\xi| \leq 1} e^{-\frac{\varepsilon}{5}|\xi|^{2-2 \theta}(t-\tau)}\left(1+|\xi|^{2(2+2 \gamma-2 \delta)}\right)\left|\widehat{u^{p}}\right|^{2} d \xi d \tau \\
& +C \int_{0}^{t} \int_{|\xi| \geq 1} e^{-\frac{\varepsilon}{10}(t-\tau)}\left(1+|\xi|^{2(2+2 \gamma-2 \delta)}\right)\left|\widehat{u^{p}}\right|^{2} d \xi d \tau
\end{aligned}
$$

On the low frequency zone we use the Lemmas 1.8 and 1.1 and on high frequency we use the Lemma 1.3 with $n<8-2 \delta$. Then, since $2+2 \gamma-2 \delta \leq$ $4-\delta$ and the condition that $\gamma \leq \frac{2+\delta}{2}$ we arrive at the estimate

$$
\begin{aligned}
N_{1} & \leq C \int_{0}^{t}\left\|u^{p}(\tau)\right\|_{L^{1}}^{2} \int_{|\xi| \leq 1} e^{-\frac{\varepsilon}{5}|\xi|^{2-2 \theta}(1+t-\tau)} d \xi d \tau \\
& +C \int_{0}^{t} e^{-\frac{\varepsilon}{10}(t-\tau)} \int_{|\xi| \geq 1}\left(1+|\xi|^{2(2+2 \gamma-2 \delta)}\right)\left|\widehat{u^{p}}(\tau)\right|^{2} d \xi d \tau \\
& \leq C \int_{0}^{t}\left\|u^{p}(\tau)\right\|_{L^{1}}^{2}(1+t-\tau)^{-\frac{n}{2-2 \theta}} d \tau+C \int_{0}^{t} e^{-\frac{\varepsilon}{10}(1+t-\tau)}\left\|u^{p}(\tau)\right\|_{H^{4-\delta}}^{2} d \tau .
\end{aligned}
$$

Now, using the Lemmas 1.3 and 1.4 with $n<8-2 \delta$ and $p>1$ integer, we have estimates to $L^{1}$-norm and $H^{A-\delta}$-norm of $u^{p}$. Thus the estimate for $N_{1}$ is obtained as

$$
\begin{aligned}
N_{1} & \leq C \int_{0}^{t}\|u\|_{H^{4-\delta}}^{2 p}(1+t-\tau)^{-\frac{n}{2-2 \theta}} d \tau+C \int_{0}^{t} e^{-\frac{\varepsilon}{10}(1+t-\tau)}\|u\|_{H^{4-\delta}}^{2 p} d \tau \\
& \leq C \int_{0}^{t}\|u\|_{H^{4-\delta}}^{2 p}(1+t-\tau)^{-\frac{n}{2-2 \theta}} d \tau, \quad \forall t>0 .
\end{aligned}
$$

Therefore, combining the above estimates, we conclude for $p>1$ integer and $n<8-2 \delta$ 


$$
\begin{aligned}
& \left\|\left(u, u_{t}\right)\right\|_{H^{4-\delta} \times H^{2}}^{2} \leq C\left(\left\|u_{0}\right\|_{L^{1}}^{2}+\left\|u_{0}\right\|_{H^{4-\delta}}^{2}\right)(1+t)^{-\frac{n}{2-2 \theta}} \\
& +C\left(\left\|u_{1}\right\|_{L^{1}}^{2}+\left\|u_{1}\right\|_{H^{2}}^{2}+\left\|u_{1}\right\|_{\dot{W}^{-1,1}}^{2}\right)(1+t)^{-\frac{n}{2-2 \theta}}+C \int_{0}^{t}\|u\|_{H^{4-\delta}}^{2 p}(1+t-\tau)^{-\frac{n}{2-2 \theta}} d \tau \\
& \leq C\left(\left\|\left(u_{0}, u_{1}\right)\right\|_{L^{1} \times L^{1}}^{2}+\left\|u_{1}\right\|_{\dot{W}^{-1,1}}^{2}+\left\|\left(u_{0}, u_{1}\right)\right\|_{H^{4-\delta} \times H^{2}}^{2}\right)(1+t)^{-\frac{n}{2-2 \theta}} \\
& +C \int_{0}^{t}\left\|\left(u, u_{t}\right)\right\|_{H^{4-\delta} \times H^{2}}^{2 p}(1+t-\tau)^{-\frac{n}{2-2 \theta}} d \tau, \quad \forall t>0
\end{aligned}
$$

and the lemma is proved.

Finally we multiply the inequality in previous lemma by $(1+t)^{\frac{n}{2-2 \theta}}$ in order to get the following inequality which holds for $t>0$.

$$
\begin{aligned}
& (1+t)^{\frac{n}{2-2 \theta}}\left\|\left(u, u_{t}\right)\right\|_{H^{4-\delta} \times H^{2}}^{2} \leq C\left(\left\|\left(u_{0}, u_{1}\right)\right\|_{L^{1} \times L^{1}}^{2}+\left\|u_{1}\right\|_{W^{-1,1}}^{2}+\left\|\left(u_{0}, u_{1}\right)\right\|_{H^{4-\delta} \times H^{2}}^{2}\right) \\
& +C \int_{0}^{t}\left\|\left(u, u_{t}\right)\right\|_{H^{4-\delta} \times H^{2}}^{2 p}(1+t)^{\frac{n}{2-2 \theta}}(1+t-\tau)^{-\frac{n}{2-2 \theta}} d \tau .
\end{aligned}
$$

Now, for $t \geq 0$ we define the function

$$
M_{1}(t)=\sup _{0 \leq \tau \leq t}(1+\tau)^{\frac{n}{2-2 \theta}}\left\|\left(u(\tau), u_{t}(\tau)\right)\right\|_{H^{4-\delta} \times H^{2}}^{2}
$$

From the above inequality we have

$$
\begin{aligned}
M_{1}(t) & \leq C\left(\left\|\left(u_{0}, u_{1}\right)\right\|_{L^{1} \times L^{1}}^{2}+\left\|u_{1}\right\|_{\dot{W}^{-1,1}}^{2}+\left\|\left(u_{0}, u_{1}\right)\right\|_{H^{4-\delta} \times H^{2}}^{2}\right) \\
& +C M_{1}(t)^{p} \int_{0}^{t}(1+\tau)^{-\frac{n p}{2-2 \theta}}(1+t)^{\frac{n}{2-2 \theta}}(1+t-\tau)^{-\frac{n}{2-2 \theta}} d \tau,
\end{aligned}
$$

for all $t>0$. By Lemma 1.9 we have

$$
\int_{0}^{t}(1+\tau)^{-\frac{n p}{2-2 \theta}}(1+t)^{\frac{n}{2-2 \theta}}(1+t-\tau)^{-\frac{n}{2-2 \theta}} d \tau \leq C(n, p, \theta)
$$

when $\frac{n}{2-2 \theta}>1$, that is, $2-2 \theta<n$ with $C(n, p, \theta)$ a positive constant.

Therefore, we have arrived at the following main inequality with holds for $t>0$. 


$$
M_{1}(t) \leq C\left(\left\|\left(u_{0}, u_{1}\right)\right\|_{L^{1} \times L^{1}}^{2}+\left\|u_{1}\right\|_{\dot{W}^{-1,1}}^{2}+\left\|\left(u_{0}, u_{1}\right)\right\|_{H^{4-\delta} \times H^{2}}^{2}\right)+C M_{1}(t)^{p} .
$$

Finally, to find the desired decay rates to the Cauchy Problem (19) we need an elementary lemma of calculus, analogous to Lemma 4.5.

Lemma 5.2: Let $p>1$ and $F(M)=a I_{0}+b M p-M$, a continuous and positive function for $M \geq 0$, and $a, b$, I0 positive constants. Then, there exist only one $M_{0}>0$ absolute minimum point of $F(M)$ in $[0, \infty)$. In addition, there exist $\varepsilon>0$ such that if $0<I_{0} \leq \varepsilon$ then $F\left(M_{0}\right)<0$.

Combining (30), the above lemma and work as in subsection 4.2 we can prove the following theorem.

Theorem 5.1: Let $0 \leq \delta \leq \theta, 0 \leq \theta \leq \frac{1}{2}, \frac{1}{2} \leq \gamma \leq \frac{2+\delta}{2}, p>1$ integer and $2-2 \theta<n<8-2 \delta$. Consider the initial data $u_{0} \in H^{4-\delta}\left(\mathrm{R}^{n}\right) \cap L^{1}\left(\mathrm{R}^{n}\right)$ and $u_{1} \in H^{2}\left(\mathrm{R}^{n}\right) \cap L^{1}\left(\mathrm{R}^{n}\right) \cap \dot{W}^{-1,1}\left(\mathrm{R}^{n}\right)$ satisfying $0<I_{0} \varepsilon$ and $M_{1}(0)<M_{0}$ with $\varepsilon$, $I_{0}$, $M_{0}, M_{1}(0)$ given by Lemma 5.2. Then the following estimate for the energy norm plus the $L^{2}$ standard of the solution is true

$$
\int_{\mathbb{R}^{n}}\left(\left|u_{t}\right|^{2}+\left|(-\Delta)^{\delta / 2} u_{t}\right|^{2}+\alpha|\Delta u|^{2}+\left|(-\Delta)^{1 / 2} u\right|^{2}+|u|^{2}\right) d x \leq C I_{0}(1+t)^{-\frac{n}{2-2 \theta}}, \quad \forall t>0 .
$$

We note here that the rate found above is the same rate found for the energy norm of Linear Problem 4 as we see in Theorem 3.3 item (i).

\subsection{Case $0 \leq \delta \leq \theta$ and $\frac{1}{2}<\theta \leq \frac{2+\delta}{2}$}

As in the previous section we prove decay rates to the energy and $L^{2}$ norm for this case. The result is the following.

Theorem 5.2: Let $0 \leq \delta \leq \theta, \frac{1}{2}<\theta \leq \frac{2+\delta}{2}, \frac{1}{2} \leq y \leq \frac{2+\delta}{2}, p>1$ integer and $2 \theta$ $<n<8-2 \delta$. Consider the initial data $u_{0} \in H^{4-\delta}\left(\mathrm{R}^{n}\right) \cap L^{1}\left(\mathrm{R}^{n}\right)$ and $u_{1} \in H^{2}\left(\mathrm{R}^{n}\right)$ $\cap L^{1}\left(R^{n}\right) \cap \dot{W}^{-1,1}\left(R^{n}\right)$ satisfying $0<I_{0} \varepsilon$ and $M_{2}(0)<M_{0}$ with $\varepsilon, I_{0}, M_{0}, M_{2}(0)$ define above. Then the following estimate for the energy norm plus the $L^{2}$ standard of the solution is true 


$$
\int_{\mathbb{R}^{n}}\left(\left|u_{t}\right|^{2}+\left|(-\Delta)^{\delta / 2} u_{t}\right|^{2}+\alpha|\Delta u|^{2}+|\nabla u|^{2}+|u|^{2}\right) d x \leq C I_{0}(1+t)^{-\frac{n}{2 \theta}}, \quad \forall t>0 .
$$

Remark 5.1: We observe that we can remove the hypotheses $\gamma \geq 1 / 2$ in Theorems 4.3, 5.1, 5.2 and assume the condition $\gamma \geq \max \{0,1 / 2-n / 4\}$ by performing a simple estimate. In fact such condition was imposed when was estimated the integral corresponding to the semilinear term in (24) and in (28) on the zone of low frequency. Indeed, we can estimate the integral with a singularity in $\xi=0$ that appears in (24).

$$
\begin{aligned}
& \left.\int_{0}^{t} \int_{|\xi| \leq 1}\left(1+|\xi|^{2(4-3 \delta)}\right)|\xi|^{4 \gamma} \frac{\left(1+|\xi|^{2 \delta}\right)}{|\xi|^{2}\left(1+\alpha|\xi|^{2}\right)} \widehat{\mid u^{p}(\tau)}\right|^{2} d \xi d \tau \leq \int_{0}^{t} \int_{|\xi| \leq 1}|\xi|^{4 \gamma-2}\left|\hat{u}^{p}(\tau)\right|^{2} d \xi d \tau \\
& \leq \int_{0}^{t}\left|u^{p}(\tau)\right|_{L^{1}}^{2} \int_{|\xi| \leq 1}|\xi|^{4 \gamma-2} d \xi d \tau \leq C \int_{0}^{t}\left|u^{p}(\tau)\right|_{L^{1}}^{2} d \tau \leq \int_{0}^{t}|u(\tau)|_{H^{4-\delta}}^{2 p} d \tau,
\end{aligned}
$$

due to Lemma 1.4 for $4-\delta>n / 2$ and the assumption $\gamma>1 / 2-n / 4$ with $\gamma>0$.

\section{REFERENCES}

CHRISTOV, C. I., MAUGIN, G. A. e VELARDE, M. G. Well-posed Boussinesq paradigm with purely spatial higher-order derivatives. Physical Review $E$, V. 54, n. 4, p. 3621-3638, 1996.

CHARÃo, R. C., DA LUZ, C. R. e IKEHATA, R. New decay rates for a problem of plate dynamics with fractional damping. Journal of Hyperbolic Differential Equations, v. 10, n. 3, p. 563-575, 2013.

DA LUZ, C. R. e CHARA O, R. C. Asymptotic properties for a semilinear plate equa- tion in unbounded domains. Journal of Hyperbolic Differential Equations, v. 6, n. 2, p. 269-294, 2009.

DA LUZ, C. R., IKEHATA, R. e CHARA O, R. C. Asymptotic behavior for abstract evolution differential equations of second order. Journal of Differential Equations, v. 259, n. 10, p. 5017-5039, 2015.

DARIPA, P. e HUA, W. A numerical study of an ill-posed Boussinesq equation arising in water waves and nonlinear lattices: Filtering and regularization techniques. Applied Mathematics and Computation, v. 101, n. 2-3, p. 159-207, 1999. 
ESFAHANI, A., FARAH, L. G. e WANG, H. Global existence and blow-up for the gen- eralized sixth-order Boussinesq equation. Nonlinear Analysis: Theory, Methods and Applications, v. 75, n. 11, p. 4325-4338, 2012.

HORBACH, J. L., IKEHATA, R. e CHARA O, R. C. Optimal Decay Rates and Asymp- totic Profile for the Plate Equation with Structural Damping. Journal of Mathe- matical Analysis and Applications, v. 440, n. 2, p. 529-560, 2016.

IKEHATA, R. e NATSUME, M. Energy decay estimates for wave equations with a fractional damping. Differential and Integral Equations, v. 25, n. 9-10, p. 939956, 2012.

IKEHATA, R. e SOGA, M. Asymptotic profiles for a strongly damped beam equa- tion with a lower order perturbation. Communications on Pure and Applied Analysis, v. 14, n. 5, p. 1759-1780, 2015.

KATO, T. e PONCE, G. Commutator estimates and the euler and navier-stokes equations. Communications on Pure and Applied Mathematics, v. 41, n. 7, p. 891-907, 1988.

MATSUMURA, A. On the asymptotic behavior of solutions of semi-linear wave equa- tions. Publications of the Research Institute for Mathematical Sciences, v. 12 , n. 1, p. 169-189, 1976-1977.

MAUGIN, G. A. Nonlinear Waves in Elastic Crystals. Oxford Science Publications, Oxford University Press, 1999.

SUGITANI, Y. e KAWASHIMA, S. Decay estimates of solutions to a semi-linear dissipative plate equation. Journal of Hyperbolic Differential Equations, v. 7, n. 3, p. 471-501, 2010.

WANG, S. e CHEN, G. Small amplitude solutions of the generalized IMBq equation. Journal of Mathematical Analysis and Applications, v. 274, n. 2, p. 846-866, 2002.

WANG, S. e CHEN, G. The Cauchy problem for the generalized IMBq equation in $W \mathrm{~s}, \mathrm{p}(\mathrm{Rn})$. Journal of Mathematical Analysis and Applications, v. 266, n. 1, p. 38-54, 2002.

WANG, S. e XUE, H. Global Solution for a Generalized Boussinesq Equation. Applied Mathematics and Computation, v. 204, n. 1, p. 130-136, 2008. 